OPEN ACCESS

Edited by:

Karsten Hiller,

Technische Universitat Braunschweig,

Germany

Reviewed by:

Johannes Meiser,

University of Glasgow

United Kingdom

Frank Pessler,

Twincore Zentrum für Experimentelle und Klinische Infektionsforschung

$\mathrm{GmbH}$, Germany

*Correspondence:

You-Dong Wei

youdongwei1966@163.com

Received: 19 January 2018

Accepted: 06 July 2018

Published: 31 August 2018

Citation:

Dong $M-X$, Feng $X, X u X-M, H u L$,

Liu Y, Jia S-Y, Li B, Chen W and

Wei Y-D (2018) Integrated Analysis

Reveals Altered Lipid and Glucose Metabolism and Identifies NOTCH2 as

a Biomarker for Parkinson's Disease

Related Depression

Front. Mol. Neurosci. 11:257.

doi: 10.3389/fnmol.2018.00257

\section{Integrated Analysis Reveals Altered Lipid and Glucose Metabolism and Identifies NOTCH2 as a Biomarker for Parkinson's Disease Related Depression}

\author{
Mei-Xue Dong ${ }^{1,2}$, Xia Feng ${ }^{3}$, Xiao-Min Xu ${ }^{1}$, Ling Hu ${ }^{4}$, Yang Liu ${ }^{1}$, Si-Yu Jia ${ }^{1}$, Bo Li ${ }^{1}$, \\ Wei Chen ${ }^{5}$ and You-Dong Wei ${ }^{1 *}$ \\ ${ }^{1}$ Department of Neurology, The First Affiliated Hospital of Chongqing Medical University, Chongqing, China, ${ }^{2}$ Department of \\ Neurology, Renmin Hospital of Wuhan University, Hubei General Hospital, Hubei, China, ${ }^{3}$ Department of Neurology, The \\ People's Hospital of Tongliang District, Chongqing, China, ${ }^{4}$ Department of Neurology, The Fifth People's Hospital of \\ Chongqing, Chongqing, China, ${ }^{5}$ Shanghai Applied Protein Technology Co., Ltd., Shanghai, China
}

Depression is a common comorbidity in Parkinson's disease (PD) but is underdiagnosed. We aim to investigate the altered metabolic pathways of Parkinson's disease-related depression (PDD) in plasma and to identify potential biomarkers for clinical diagnosis. Consecutive patients with PD were recruited, clinically assessed, and patients with PDD identified. Fasting plasma samples were collected from 99 patients and differentially expressed metabolites and proteins between patients with PDD and PD were identified using non-targeted liquid chromatography-mass spectrometry (LC-MS)-based metabolomics and tandem mass tag (TMT)-based proteomics analysis, followed by an integrated analysis. Based on the above results, enzyme-linked immune sorbent assay (ELISA) tests were then performed to identify potential biomarkers for PDD. In clinics, patients with PDD suffered less hypertension and had lower serum low-density lipoprotein cholesterol and apolipoprotein B levels when compared to the other patients with PD. A total of 85 differentially expressed metabolites were identified in metabolomics analysis. These metabolites were mainly lipids and lipid-like molecules, involved in lipid and glucose metabolic pathways. According to proteomics analysis, 17 differentially expressed proteins were identified, and 12 metabolic pathways were enriched, which were predominantly related to glucose metabolism. Integrated analysis indicated that altered lipid and glucose metabolism in PDD may induce cellular injury through oxidative stress. Additionally, plasma levels of several proteins were confirmed to be significantly altered and correlated with depressive severity. $\mathrm{NOTCH} 2$ may be a potential blood biomarker for PDD, with an optimal cut-off point of $0.91 \mathrm{ng} / \mathrm{ml}$, a sensitivity value of $95.65 \%$, and a specificity value of $81.58 \%$. Depressive symptoms are associated with lipid and glucose metabolism in patients with PD and NOTCH2 may be a potential blood biomarker for the clinical diagnosis of PDD.

Keywords: Parkinson's disease, depression, proteomics, metabolomics, integrated analysis, biomarker 


\section{INTRODUCTION}

Parkinson's disease (PD) is the second most prevalent neurodegenerative disease following Alzheimer's disease, afflicting about $3.15 \%$ of people over 40 years old (Pringsheim et al., 2014). It seriously affects the patient's quality of life, increases mortality rate, and places a heavy economic burden on the family and society (Kowal et al., 2013; Macleod et al., 2014). Although various studies have been conducted to identify diagnostic biomarkers (Sharma et al., 2013), the clinical diagnosis of patients with $\mathrm{PD}$ has been primarily based on cardinal motor features, including resting tremor, bradykinesia, and rigidity (Postuma et al., 2015). Nonmotor symptoms, such as constipation (Adams-Carr et al., 2016), hyposmia (Ponsen et al., 2009), rapid eye movement behavior disorder, cognition (Aarsland et al., 2005), anxiety (Broen et al., 2016), and depression (Reijnders et al., 2008) are also reportedly more prevalent in patients with $\mathrm{PD}$. This is probably due to the alteration of extensive neurotransmitters and brain regions in patients with PD (Kalia and Lang, 2015). Those nonmotor symptoms are often more problematic and distressing than the cardinal motor symptoms.

Depression is one of the most prevalent nonmotor symptoms in $\mathrm{PD}$ patients, with about $17 \%$ of patients with $\mathrm{PD}$ suffering from major depressive disorder (Reijnders et al., 2008). Depression can exacerbate motor symptoms, induce further cognitive disorders, deteriorate the quality of life, and increase the suicide rate in patients with PD (Gustafsson et al., 2015). Worse still, depression in patients with $\mathrm{PD}$ is underdiagnosed and undertreated, with only approximately $20 \%$ of those diagnosed actually receiving treatments (Goodarzi et al., 2016). Depression can be easily ignored as its characteristic symptoms, including loss of appetite and energy, sleep disturbances, and fatigue, overlap with other symptoms in patients with PD (Marsh, 2013). The underdiagnosis of depression in patients with PD can lead to poor outcomes for patients and caregivers. Currently, the diagnosis of depression in patients with PD is based on a clinical interview (Goodarzi et al., 2016), and an accurate and objective way is highly desirable for clinicians. In addition, treatments for the diagnosis of depression in patients with PD are based on clinical interviews (Goodarzi et al., 2016). Similarly, patients with definitive Parkinson's disease-related depression (PDD) also should be individualized and multidimensional (Marsh, 2013), while the underlying pathogenesis of PDD is complex and remains unclear (Sharma et al., 2013).

Various omics data can extend the understanding of many diseases and help us make more discoveries (Campbell et al., 2017). Novel bioinformatics methods further enable integrated perspectives of pathogeneses and identify clinical biomarkers (Berger et al., 2013; Mostafa et al., 2016). Metabolomics is a comprehensive evaluation of total endogenous metabolites while proteomics expands the understanding of a biological system at the protein level (Altelaar et al., 2013; Wishart, 2016). Herein we employed non-targeted liquid chromatography-mass spectrometry (LC-MS)-based metabolomics analysis and tandem mass tag (TMT)-based proteomics analysis to investigate the plasma changes of PDD. An integrated analysis was further generated to elucidate the probable pathogenesis. Based on the above results, enzyme-linked immune sorbent assay (ELISA) tests were then performed to identify potential biomarkers for PDD. In clinical settings, plasma is a relatively accessible, stable, and informative biofluid, making it ideal for exploring the underlying pathogenesis, and facilitating the diagnosis of PDD (Hu et al., 2016; Dong et al., 2017c).

\section{MATERIALS AND METHODS}

\section{Patients}

One hundred and ten consecutively recruited patients with PD were included in the Department of Neurology, the First Affiliated Hospital of Chongqing Medical University from April 2016 to February 2017 in accordance with the following inclusion criteria: (i) Parkinson's disease diagnosed according to the recommendation from the European Federation of Neurological Societies (EFNS) and the International Parkinson Movement Disorder Society's European Section (MDS-ES) (Berardelli et al., 2013); (ii) Patients not treated with any drugs other than dopamine analogs or dopamine receptor agonists. Eleven patients were then excluded according to the following criteria: (i) secondary Parkinson disorders or Parkinson-plus syndrome; (ii) coexistence of severe systemic diseases (e.g., tumor, chronic heart failure, chronic obstructive pulmonary disease, hepatitis, and nephritis) or infectious diseases at the time of enrolment; (iii) history of stroke, brain surgery or injury, Alzheimer's disease, motor neuron disease, or other diseases in the central nervous system (Dong et al., 2017c).

This study was approved by the ethics committee of the First Affiliated Hospital of Chongqing Medical University and performed in accordance with the Declaration of Helsinki. All subjects provided written informed consent prior to inclusion in this study. Clinical characteristics, metabolomics analysis, and proteomics analysis were blindly assessed or performed.

\section{Clinical Characteristics}

The clinical characteristics of all included patients were recorded. Fasting plasma samples were obtained by centrifugation at $2,000 \times g$ for $10 \mathrm{~min}$ at $4^{\circ} \mathrm{C}$ after collection with EDTAK2 tube at 6:00 a.m. by puncture of the median cubital vein. Samples were then stored at $-80^{\circ} \mathrm{C}$ until assay. All patients were interviewed by experienced physicians and patients with PDD were diagnosed according to Diagnostic and Statistical Manual of Mental Disorders (DSM-IV) (Starkstein et al., 2008), with Hamilton depression scores $>17$ on the 17 -item Hamilton Rating Scale for Depression (HAMD) (Dissanayaka et al., 2007). All scales were independently assessed by two physicians.

\section{Metabolomics Analysis}

Liquid chromatography-mass spectrometry (LC-MS)-based metabolomics of plasma samples (23 patients with PDD and 40 randomly selected patients with PD) were performed on a Waters UPLC I-class system equipped with a binary solvent delivery manager (Waters Corporation, Milford, USA) (Zhang et al., 2017a). 
Plasma samples stored at $-80^{\circ} \mathrm{C}$ were gradually thawed on ice, 2-chloro-1-phenylalanine dissolved in methanol $(0.3 \mathrm{mg} / \mathrm{mL})$ was served as internal standard. In a $1.5 \mathrm{~mL}$ Eppendorf tube, $50 \mu \mathrm{L}$ of sample and $10 \mu \mathrm{L}$ of internal standard were added and then vortexed for $10 \mathrm{~s}$. Subsequently, $150 \mu \mathrm{L}$ of ice-cold mixture of methanol and acetonitrile (2/1, vol/vol) were added. The mixtures were vortexed for $1 \mathrm{~min}$, ultrasonicated at ambient temperature $\left(25^{\circ} \mathrm{C}\right)$ for $5 \mathrm{~min}$, placed at $-20^{\circ} \mathrm{C}$ for $10 \mathrm{~min}$, and centrifuged at $15,000 \mathrm{rpm}$ at $4^{\circ} \mathrm{C}$ for $10 \mathrm{~min}$. $100 \mu \mathrm{L}$ of the supernatants from each tube were collected, filtered through $0.22 \mu \mathrm{m}$ microfilters, and transferred to LC vials. The vials were stored at $-80^{\circ} \mathrm{C}$ until LC-MS analysis. Quality control sample was obtained by mixing all the samples equally as a pooled sample, and then processed using the above method with the analytic reagents. The quality control samples were injected at regular intervals (every 10 samples) throughout the analytical run to provide a set of data from which repeatability can be assessed.

Acquity BEH C18 column $(100 \times 2.1 \mathrm{~mm}$ i.d., $1.7 \mu \mathrm{m}$; Waters, Milford, USA) was engaged and maintained at $45^{\circ} \mathrm{C}$. The following gradients were used for separation: 5-20\% B over 0-2 min, $20-60 \%$ B over $2-8 \mathrm{~min}, 60-100 \%$ B over $8-12 \mathrm{~min}$, $100 \%$ B for $2 \mathrm{~min}, 100-5 \%$ Bover $14-14.5 \mathrm{~min}$, and $14.5-15.5 \mathrm{~min}$ holding at $5 \% \mathrm{~B}$ at a flow rate of $0.40 \mathrm{~mL} / \mathrm{min}$, where $\mathrm{B}$ is acetonitrile $(0.1 \%(\mathrm{v} / \mathrm{v})$ formic acid) and $\mathrm{A}$ is aqueous formic acid $[0.1 \%(\mathrm{v} / \mathrm{v})$ formic acid]. Injection volume was $3.00 \mu \mathrm{L}$ and column temperature was set at $45^{\circ} \mathrm{C}$.

The mass spectrometric data was collected using a Waters VION IMS Q-TOF Mass Spectrometer equipped with an electrospray ionization (ESI) source operating in either positive or negative ion mode. The source temperature and desolvation temperature were set at $120^{\circ} \mathrm{C}$ and $500^{\circ} \mathrm{C}$, respectively, with a desolvation gas flow of $900 \mathrm{~L} / \mathrm{h}$. Centroid data were collected from 50 to $1,000 \mathrm{~m} / \mathrm{z}$ with a scan time of $0.1 \mathrm{~s}$ and interscan delay of $0.02 \mathrm{~s}$ over a $13 \mathrm{~min}$ analysis time. The UPLC-Q-TOF/MS raw data were analyzed by progenesis QI software (Waters Corporation, Milford, USA) using the following parameters. Retention time (RT) ranged from 0.5 to $14.0 \mathrm{~min}$, mass ranged from 50 to $1,000 \mathrm{Da}$, and mass tolerance was $0.01 \mathrm{Da}$. Isotopic peaks were excluded for analysis, noise elimination level was set at 10.00 , minimum intensity was set to $15 \%$ of base peak intensity, and RT tolerance was set at $0.01 \mathrm{~min}$. Three-dimension data sets including $\mathrm{m} / \mathrm{z}$, peak RT, and peak intensities were exported into an Excel file, and $\mathrm{RT}-\mathrm{m} / \mathrm{z}$ pairs were used to identify each ion based on Human Metabolome Database (HMDB, http://www.hmdb.ca), Metlin (https://metlin.scripps.edu), and LipidMaps (http://www.lipidmaps.org). The resulting matrix was further reduced by removing any peaks with missing values (ion intensity $=0$ ) in more than $60 \%$ of samples. The internal standard was used for data quality control.

The positive and negative data were combined into a data set and imported into SIMCA-P+ 13.0 software package (Umetrics, Umeå, Sweden) for multivariate statistical analysis. An orthogonal partial least squares-discriminant analysis (OPLSDA) model was used to exhibit statistical differences and identify differentially expressed metabolites in patients with PDD relative to the other patients with $\mathrm{PD}$, and this model was validated by a permutation test with 200 iterations. The differentially expressed metabolites were recognized with variable influence on projection values of greater than 1.0, fold change values of greater than \pm 1.5 , and $p$-values (from Mann-Whitney $U$-tests) of less than 0.05 (Zheng et al., 2016; Dong et al., 2017b). The classifications of differentially expressed metabolites were based on the HMDB database. Metabolic pathway overrepresentation enrichment analysis was also performed using Integrated Molecular Pathway Level Analysis (http://impala.molgen.mpg.de) (Kamburov et al., 2011). $Q$ values were calculated by Benjamini-Hochberg correction for multiple testing to adjust the derived $p$ values and significances were considered at $q$-values $<0.05$ (Hochberg and Benjamini, 1990).

\section{Proteomics Analysis}

The details of this process have been described previously (Dayon et al., 2014; Sogawa et al., 2016). Briefly, six pooled samples were obtained by the accumulation of $50 \mu \mathrm{l}$ of each plasma sample (15 randomized patients with PDD and 30 randomized patients with $\mathrm{PD}$ were equally grouped into three pooled samples, respectively). Most of the abundant proteins were depleted from those pooled samples using Agilent Human 14 Multiple Affinity Removal System Column (Agilent Technologies, California, USA) following the manufacturer's protocol. The $10 \mathrm{KDa}$ ultrafiltration tube (Sartorius, Göttingen, Germany) was used for desalination and concentration of the low-abundance components. One volume of SDT buffer (4\% SDS, $100 \mathrm{mM}$ Tris-HCl, $1 \mathrm{mM}$ DTT, $\mathrm{pH} 7.6)$ was added. The mixture was boiled for $15 \mathrm{~min}$ and centrifuged at $14,000 \mathrm{~g}$ for 20 min. The supernatant was quantified with the BCA Protein Assay Kit (Bio-Rad, California, USA). Finally, the sample was stored at $-80^{\circ} \mathrm{C}$ until assay.

For each sample, $20 \mu \mathrm{g}$ of proteins were mixed with $5 \mathrm{X}$ loading buffer and boiled for $5 \mathrm{~min}$. The proteins were then separated on $12.5 \%$ SDS-PAGE gel (constant current $14 \mathrm{~mA}$, $90 \mathrm{~min}$ ) and protein bands were visualized by Coomassie Blue R-250 staining for quality control.

After that, $200 \mu \mathrm{g}$ of proteins for each sample were incorporated into $30 \mu \mathrm{l}$ SDT buffer (4\% SDS, $100 \mathrm{mM}$ DTT, $150 \mathrm{mM}$ Tris- $\mathrm{HCl} \mathrm{pH}$ 8.0). The detergent, dithiothreitol (DTT), and other low-molecular-weight components were removed using UA buffer ( $8 \mathrm{M}$ Urea, $150 \mathrm{mM}$ Tris- $\mathrm{HCl} \mathrm{pH} \mathrm{8.0)} \mathrm{by}$ repeated ultrafiltration. Then $100 \mu \mathrm{l}$ iodoacetamide $(100 \mathrm{mM}$ IAA in UA buffer) was added to block reduced cysteine residues and the samples were incubated for $30 \mathrm{~min}$ in darkness. The filters were washed with $100 \mu \mathrm{l}$ UA buffer three times and then $100 \mu \mathrm{l} 100 \mathrm{mM}$ TEAB buffer twice. Finally, the protein suspensions were digested with $4 \mu \mathrm{g}$ trypsin (Promega, Wisconsin, USA) in $40 \mu \mathrm{l} \mathrm{TEAB}$ buffer overnight at $37^{\circ} \mathrm{C}$, and the resulting peptides were collected as a filtrate. The peptide content was estimated by ultraviolet light $(280 \mathrm{~nm})$ using an extinction coefficient of 1.1 of $0.1 \%(\mathrm{~g} / \mathrm{l})$ solution that was calculated based on the frequency of tryptophan and tyrosine in vertebrate proteins.

Using TMT reagents according to the manufacturer's instructions, $100 \mu \mathrm{g}$ peptide mixture of each sample was labeled (Thermo Scientific, Massachusetts, USA). A Pierce high $\mathrm{pH}$ reverse-phase fractionation kit (Thermo Scientific) was used to fractionate TMT-labeled digest samples into 15 fractions by an 
increasing acetonitrile step-gradient elution carried according to the instructions.

Each fraction was loaded onto a reverse phase trap column connected to the C18-reversed phase analytical column in buffer A ( $0.1 \%$ Formic acid) and separated with a linear gradient of buffer B ( $84 \%$ acetonitrile and $0.1 \%$ Formic acid) at a flow rate of $300 \mathrm{nl} / \mathrm{min}$ controlled by IntelliFlow technology (Thermo Scientific) for nano LC-MS/MS analysis.

Liquid chromatography-mass spectrometry/MS (LC-MS/MS) analysis was performed on a Q Exactive mass spectrometer (Thermo Scientific) that was coupled to Easy nLC for $60 \mathrm{~min}$. The mass spectrometer was operated in positive ion mode. Mass spectrometric data was acquired using a data-dependent top 10 method dynamically by choosing the most abundant precursor ions from the survey scan $(300-1,800 \mathrm{~m} / \mathrm{z})$ for HCD fragmentation. Automatic gain control (AGC) target was set to 3E6 and maximum inject time to $10 \mathrm{~min}$. Dynamic exclusion duration was $40 \mathrm{~s}$. Survey scans were acquired at a resolution of 70,000 at $200 \mathrm{~m} / \mathrm{z}$ and resolution for HCD spectra was set to 35,000 at $200 \mathrm{~m} / \mathrm{z}$, and isolation width was $2 \mathrm{~m} / \mathrm{z}$. Normalized collision energy was $30 \mathrm{eV}$ and the underfill ratio, which specifies the minimum percentage of the target value likely to be reached at maximum fill time, was defined as $0.1 \%$. The instrument was run with the peptide recognition mode enabled.

Tandem mass spectrometry (MS/MS) spectra were searched using MASCOT engine (Matrix Science, London, UK; version 2.2) embedded into Proteome Discoverer 1.4. The differentially expressed proteins were identified by fold change values of greater than \pm 1.2 and $p<0.05$ (from Mann-Whitney $U$ tests). Gene ontology (GO) enrichment on three ontologies (biological process, molecular function, and cellular component) and Kyoto Encyclopedia of Genes and Genomes (KEGG) pathway enrichment analyses were applied based on the Fisher's exact test, considering the whole quantified protein annotations as background dataset. Benjamini-Hochberg correction for multiple testing was further applied to adjust derived $p$ values and significances were considered at $q$-values $<0.05$ (Hochberg and Benjamini, 1990).

\section{Integrated Analysis}

To explore the metabolic pathways associated with the differentially expressed metabolites and proteins, we used the commercially obtained Ingenuity Pathway Analysis software (IPA, QIAGEN, Düsseldorf, Germany) to annotate enriched molecular or cellular functions and to generate metaboliteprotein integrated networks (Dong et al., 2017b; Shen et al., 2017). We uploaded the lists and fold change values of differentially expressed metabolites and proteins onto the IPA software. Significant molecular or cellular functions were automatically annotated $(p<0.05)$ and enriched into several categories. The software also computed a $p$ score for each of the possible networks in accordance with the fit homology to all the input molecules. This score is derived from a $p$ value and indicates the probability of the input molecules in a given network to coexist as a result of random chance $\left[p\right.$ score $=-\log _{10}$ ( $p$ value)].

\section{ELISA Tests}

According to the above network from integrated analysis, plasma levels of six differentially expressed proteins [receptortype tyrosine-protein phosphatase zeta (PTPRZ1), major histocompatibility complex class I antigen (HLA-A), neurogenic locus notch homolog protein 2 (NOTCH2), lipoprotein (LPA), L-lactate dehydrogenase A chain (LDHA), and glyceraldehyde3-phosphate dehydrogenase (GAPDH)] identified by proteomics analysis were further confirmed in 23 patients with PDD and 76 patients with $\mathrm{PD}$, using ELISA kits obtained from Cloud Clone Corp (Texas, USA) according to the manufacturer's instructions (Hu et al., 2016).

\section{Statistical Analysis}

Statistical analyses were completed using a commercially available software package (IBM SPSS version 22.0, New York, USA), with statistical diagrams produced using GraphPad Prism 5 (GraphPad Software, California, USA) (Dong et al., $2017 \mathrm{a}, \mathrm{c})$. Continuous data were expressed as mean \pm standard errors of the mean and compared using Mann-Whitney $U$ tests. Categorical data were exhibited as absolute numbers and

TABLE 1 | Clinical characteristics of all patients with PD with and without depression included in this study.

\begin{tabular}{|c|c|c|c|c|c|c|c|}
\hline Variable (SEM/\%) & PD (76) & PDD (23) & $p$ value & Variable (SEM/\%) & PD (76) & PDD (23) & $p$ value \\
\hline Age (year) & $68.92 \pm 1.04$ & $66.48 \pm 2.16$ & 0.273 & RBG (mmol/L) & $7.01 \pm 0.24$ & $7.28 \pm 0.62$ & 0.623 \\
\hline Gender, Male (\%) & 44 (57.9\%) & $10(43.5 \%)$ & 0.224 & $\mathrm{HbA1C}(\%)$ & $5.97 \pm 0.09 \%$ & $7.09 \pm 0.85 \%$ & 0.230 \\
\hline Smoking history (\%) & 15 (19.7\%) & $2(8.7 \%)$ & 0.360 & $\mathrm{TC}(\mathrm{mmol} / \mathrm{L})$ & $3.95 \pm 0.18$ & $3.70 \pm 0.29$ & 0.511 \\
\hline Alcohol consumption (\%) & $7(9.2 \%)$ & $1(4.3 \%)$ & 0.754 & $\mathrm{TG}(\mathrm{mmol} / \mathrm{L})$ & $1.24 \pm 0.09$ & $1.12 \pm 0.16$ & 0.498 \\
\hline Hypertension (\%) & $31(40.8 \%)$ & $4(17.4 \%)$ & 0.040 & HDL-C (mmol/L) & $1.38 \pm 0.05$ & $1.49 \pm 0.07$ & 0.261 \\
\hline Diabetes mellitus (\%) & $9(11.8 \%)$ & $4(17.4 \%)$ & 0.735 & LDL-C (mmol/L) & $2.78 \pm 0.09$ & $2.37 \pm 0.15$ & 0.032 \\
\hline Hypercholesterolemia (\%) & $16(21.1 \%)$ & $2(8.7 \%)$ & 0.299 & Apo-A1 (g/L) & $1.33 \pm 0.03$ & $1.38 \pm 0.04$ & 0.422 \\
\hline $\mathrm{CHD}(\%)$ & $12(15.8 \%)$ & $4(17.4 \%)$ & 1.000 & Apo-B (g/L) & $0.88 \pm 0.03$ & $0.74 \pm 0.04$ & 0.010 \\
\hline Disease duration (year) & $5.62 \pm 0.63$ & $7.35 \pm 0.93$ & 0.163 & HAMD score & $8.96 \pm 0.61$ & $21.48 \pm 0.57$ & 0.000 \\
\hline $\mathrm{BMl}\left(\mathrm{kg} / \mathrm{m}^{2}\right)$ & $23.11 \pm 0.46$ & $22.25 \pm 0.67$ & 0.340 & & & & \\
\hline
\end{tabular}

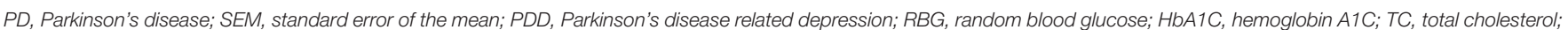

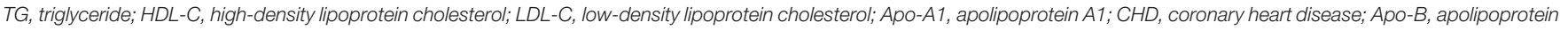
B; HAMD, Hamilton Depression Scale; BMI, body mass index. 
percentages (\%) and analyzed using Pearson $\chi^{2}$-tests or Fisher exact tests. Pearson correlation analysis between plasma protein levels and HAMD scores were performed and plotted using Cytoscape software 3.4.0 (Zhao et al., 2017). Receiver operating characteristic (ROC) curves were plotted, the optimal cut-off points were determined, and diagnostic efficacies were compared using MedCalc statistical software 15.2.2 (Ostend, Belgium). Pvalues $<0.05$ were considered significant (Dong et al., 2016).

\section{RESULTS}

\section{Clinical Characteristics}

A total of 99 patients with PD were finally included after the exclusion of 11 patients. Depressive symptoms were present in 23 patients $(23.2 \%)$, which was approximately equal to the reported $17 \%$ in the literature (Reijnders et al., 2008). The clinical characteristics of the patients with PD are shown in Table 1. The 17-item HAMD scores of patients with PDD were significantly higher than those in patients with PD. Patients with PDD also suffered less hypertension and had lower serum low-density lipoprotein cholesterol (LDL-C) and apolipoprotein B (ApoB) levels than the patients with PD. There were no significant differences in any other clinical characteristics, including the levels of blood glucose and hemoglobin $\mathrm{AlC}(\mathrm{HbA1C})$ and incidence of diabetes mellitus.

\section{Metabolomics Analysis}

The clinical characteristics of the patients included in the LC-MS-based metabolomics analysis are shown in Supplementary Table 1 and the results were comparable

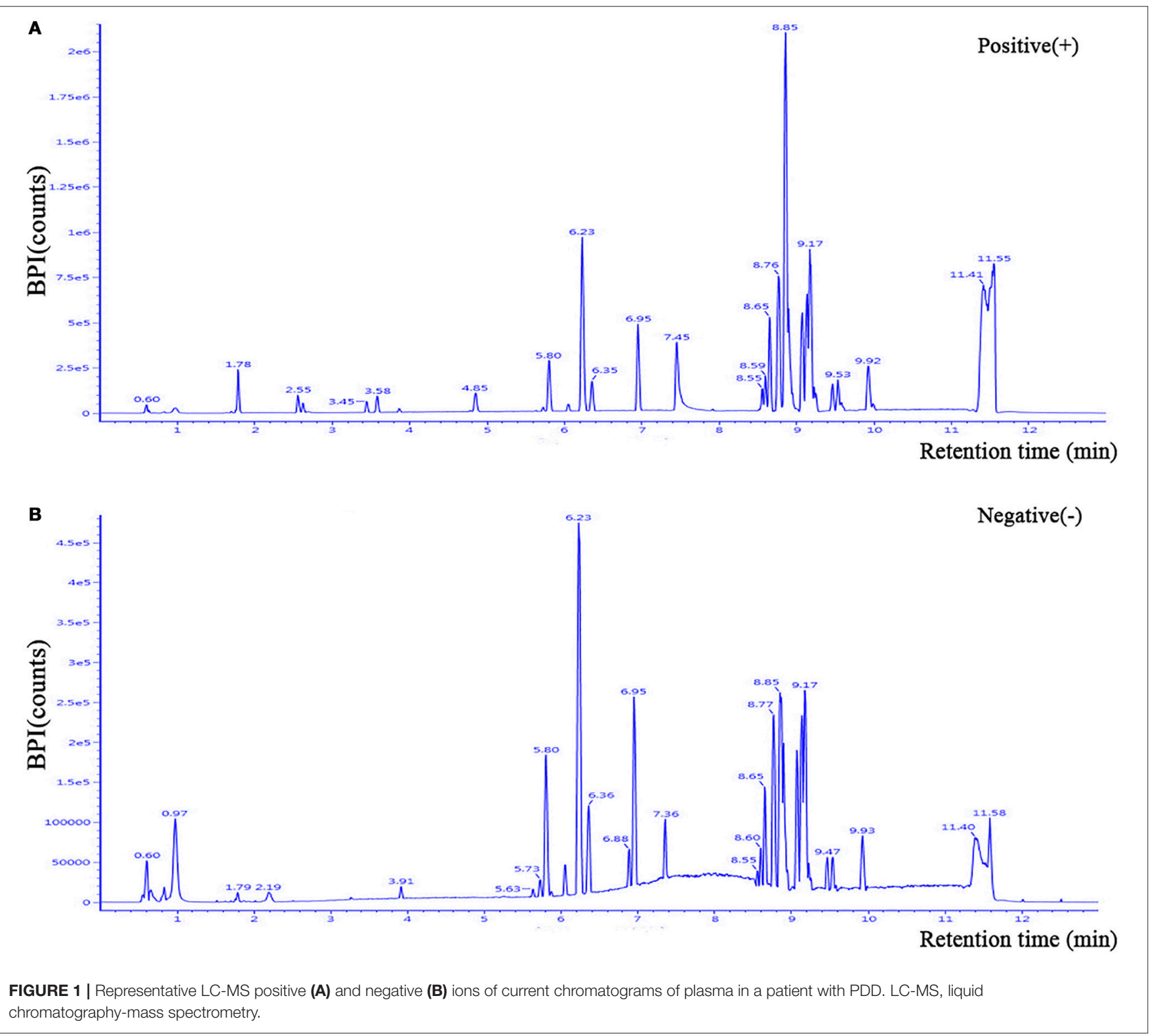


with the total patient group. Representative LC-MS positive and negative ions of current chromatograms are shown in Figure 1. After excluding internal standards, a total of 10403 individual peaks, including 6040 positive and 4363 negative peaks, were detected in approximately $98.8 \%$ of samples in each group. Based on these peaks, orthogonal partial least squares-discriminant analysis (OPLS-DA) was performed and the result showed a clear separation between the two groups $\left(\mathrm{R}^{2} \mathrm{X}=0.389, \mathrm{R}^{2} \mathrm{Y}=0.832\right.$, and $Q^{2}=0.206$ ) (Figure 2A). Moreover, a permutation test with 200 iterations confirmed that the constructed OPLS-DA model was valid and not over-fitted, as the original right $R^{2}$ and
$Q^{2}$ values were significantly higher than the corresponding permutated left values $\left[R^{2}=(0.0,0.443), Q^{2}=(0.0,-0.153)\right.$; Figure 2B].

Eighty-five differentially expressed metabolites were identified between the two groups (Table 2). Of these $75 \%$ were lipids and lipid-like molecules, $8 \%$ were organic acids and derivatives, $6 \%$ were organic oxygen compounds, $3 \%$ were organoheterocyclic compounds, and $2 \%$ were tetrapyrroles and derivatives (Figure 2C). Carbohydrates and carbohydrate conjugates were a subclass of organic oxygen compounds and contained four differentially expressed metabolites ([6]gingerdiol 4'-O-beta-D-glucopyranoside, ribitol, galactan, and

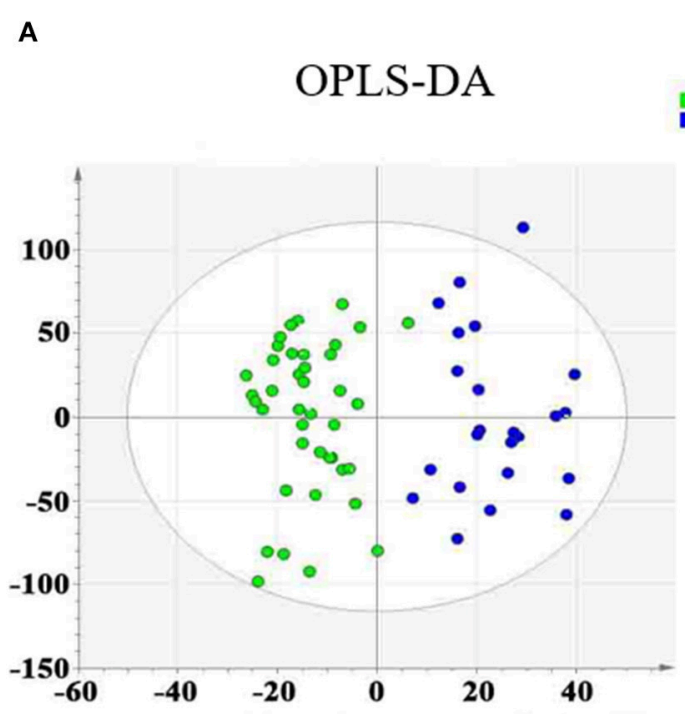

C

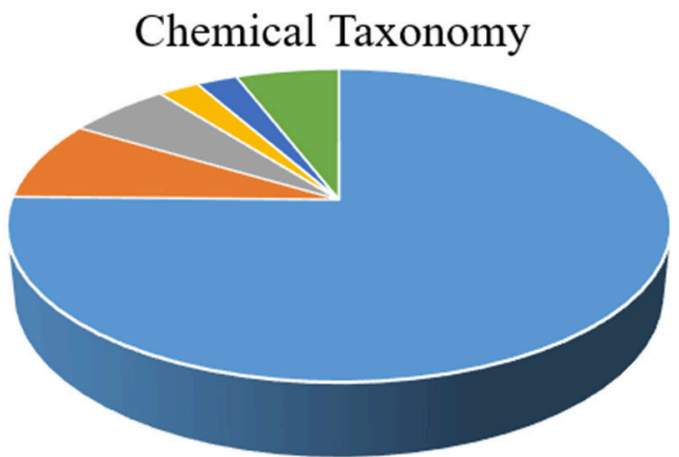

- Lipids and lipid-like molecules 75\%

- Organic acids and derivatives $8 \%$

- Organic oxygen compounds 6\%

" Organoheterocyclic compounds 3\%

- Tetrapyrroles and derivatives $2 \%$

- Others $6 \%$
B

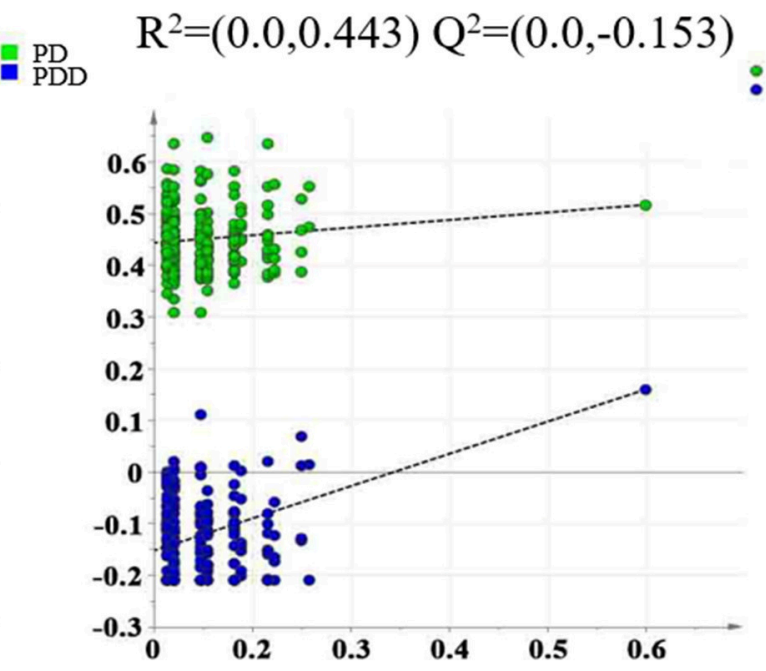

D

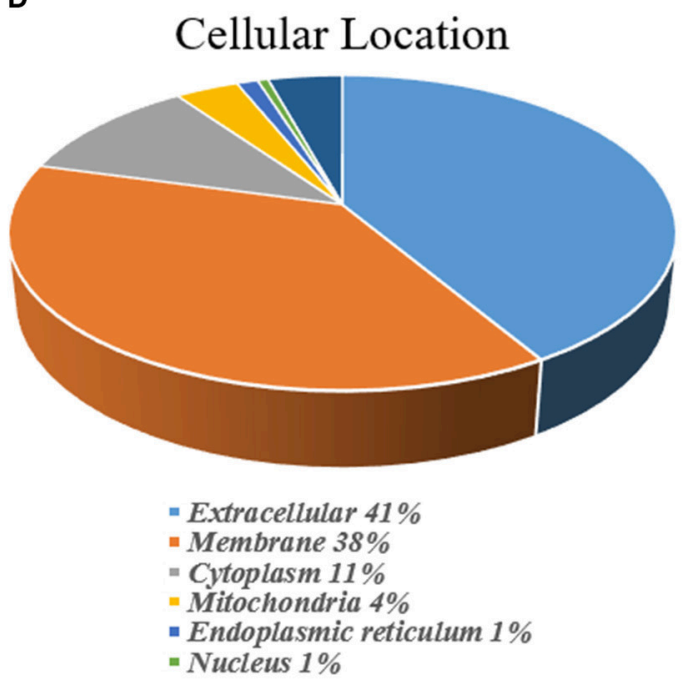

FIGURE 2 | Multivariate statistical analysis of metabolomics and classification of differentially expressed metabolites between patients with PDD and PD. (A) OPLS-DA score plot derived from liquid chromatography-mass spectrometry-based metabolomics analysis of patients with PDD (blue circles) and patients with PD (green circles). (B) Statistical validation of the OPLS-DA model by permutation testing. Representations of metabolites in terms of (C) chemical taxonomy and (D) cellular locations, based on the annotations of Human Metabolome Database. OPLS-DA, orthogonal partial least squares-discriminant analysis; PDD, Parkinson's disease-related depression; PD, Parkinson's disease. 
TABLE 2 | Key differentially expressed metabolites identified by liquid chromatography-mass spectrometry-based metabolomics analysis between patients with PD with and without depression.

\begin{tabular}{|c|c|c|c|c|c|c|c|}
\hline Compound ID & Compound name & $\mathrm{m} / \mathrm{z}$ value & RT (min) & Ion mode & FCvalue $^{b, c}$ & VIPvalue $^{c}$ & pvalue ${ }^{c}$ \\
\hline HMDB36133 & $\begin{array}{l}\text { 3-[[5-methyl-2-(1-methylethyl)cyclohexyl] oxy]-1,2- } \\
\text { propanediol }\end{array}$ & 275.1573 & 6.6012 & Positive & 0.6558 & 1.7468 & 0.0000 \\
\hline HMDB31892 & Capsianoside II & 362.5188 & 6.2251 & Positive & 0.6412 & 1.1183 & 0.0011 \\
\hline HMDB02327 & 1,11-undecanedicarboxylic acid & 511.3255 & 6.2251 & Positive & 0.6114 & 1.3346 & 0.0030 \\
\hline HMDB56318 & DG(20:2n6/0:0/20:5n3) & 349.2424 & 7.8697 & Positive & 2.4783 & 1.1013 & 0.0038 \\
\hline HMDB02472 & $\begin{array}{l}\text { (3a,5b,7a,12a)-24-[(carboxymethyl)amino]-1,12- } \\
\text { dihydroxy-24-oxocholan-3-yl-b-D- } \\
\text { glucopyranosiduronic a }\end{array}$ & 622.3352 & 6.4951 & Negative & 0.5919 & 1.3399 & 0.0038 \\
\hline HMDB09112 & $\mathrm{PE}(18: 2(9 Z, 12 Z) / 24: 0)$ & 291.6477 & 6.7939 & Positive & 0.5684 & 1.2768 & 0.0039 \\
\hline HMDB11768 & Cer(d18:0/24:0) & 564.3164 & 6.3557 & Positive & 0.6219 & 1.1326 & 0.0043 \\
\hline HMDB12052 & Ganglioside GT3 & 849.9375 & 6.2251 & Positive & 0.5873 & 1.0936 & 0.0046 \\
\hline HMDB02231 & Eicosenoic acid & 309.2803 & 7.8649 & Negative & 1.7393 & 1.1741 & 0.0053 \\
\hline 5283546 & Ubiquinone 8 & 707.5316 & 9.3385 & Negative & 0.5691 & 1.5228 & 0.0055 \\
\hline HMDB61687 & Estradiol acetate glucuronide & 491.2330 & 7.8697 & Positive & 2.1162 & 1.0804 & 0.0062 \\
\hline HMDB07004 & $\mathrm{CPA}(18: 0)$ & 457.2138 & 7.8649 & Negative & 1.7405 & 1.3792 & 0.0066 \\
\hline HMDB05050 & $15 \mathrm{H}-11,12-\mathrm{EETA}$ & 717.4415 & 9.3385 & Negative & 0.6480 & 1.3399 & 0.0067 \\
\hline HMDB35884 & Melledonol & 435.2033 & 7.8697 & Positive & 1.8629 & 1.1410 & 0.0070 \\
\hline HMDB10393 & LysoPC(20:3(5Z,8Z,11Z)) & 580.3185 & 6.0620 & Negative & 0.6378 & 1.1149 & 0.0072 \\
\hline HMDB00054 & Bilirubin & 602.2940 & 6.9452 & Positive & 0.6526 & 1.1481 & 0.0072 \\
\hline HMDB61112 & 3-carboxy-4-methyl-5-propyl-2-furanpropionic acid & 503.1898 & 7.8697 & Positive & 1.6347 & 1.1415 & 0.0073 \\
\hline HMDB06528 & Docosapentaenoic acid & 329.2489 & 7.0345 & Negative & 1.5535 & 1.2833 & 0.0079 \\
\hline HMDB13132 & Hydroxyvalerylcarnitine & 571.3428 & 6.6101 & Negative & 0.6668 & 1.5142 & 0.0085 \\
\hline HMDB36122 & [6]-gingerdiol 4'-O-beta-D-glucopyranoside & 439.2376 & 7.8649 & Negative & 2.3428 & 1.3651 & 0.0092 \\
\hline HMDB10357 & Tetrahydroaldosterone-3-glucuronide & 539.2509 & 2.8699 & Negative & 0.5838 & 1.4905 & 0.0098 \\
\hline HMDB03876 & 15(S)-hydroxyeicosatrienoic acid & 367.2137 & 7.8628 & Positive & 1.6883 & 1.2439 & 0.0099 \\
\hline HMDB03178 & Heme & 661.1760 & 7.8718 & Negative & 2.2621 & 1.4164 & 0.0101 \\
\hline HMDB04924 & Ganglioside GD2 (d18:0/18:1(9Z)) & 856.9514 & 6.2251 & Positive & 0.5699 & 1.3039 & 0.0101 \\
\hline HMDB33372 & Stearoyllactic acid & 377.2690 & 7.8649 & Negative & 1.6552 & 1.2258 & 0.0101 \\
\hline HMDB04666 & 2-arachidonylglycerol & 417.2318 & 7.8628 & Positive & 1.6767 & 1.0197 & 0.0111 \\
\hline HMDB00207 & Cis-cetoleic acid & 337.3120 & 8.3029 & Negative & 2.0741 & 1.7107 & 0.0126 \\
\hline HMDB40733 & Phytyl acetate & 383.2901 & 8.3010 & Positive & 2.6419 & 1.6090 & 0.0144 \\
\hline HMDB01181 & 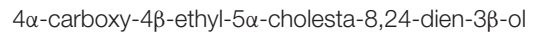 & 519.2643 & 8.3010 & Positive & 2.6728 & 1.6980 & 0.0145 \\
\hline HMDB02385 & Celastrol & 485.2448 & 8.3029 & Negative & 2.3661 & 1.5956 & 0.0155 \\
\hline HMDB00235 & Thiamine & 531.2198 & 8.3010 & Positive & 2.3720 & 1.6565 & 0.0157 \\
\hline HMDB06765 & 2-methoxy-estradiol-17b 3-glucuronide & 459.2057 & 7.8718 & Negative & 1.5666 & 1.1844 & 0.0157 \\
\hline HMDB33337 & Fusarin $\mathrm{C}$ & 468.1464 & 6.4264 & Negative & 1.7382 & 1.4644 & 0.0168 \\
\hline HMDB00809 & N-glycoloylganglioside GM1 & 812.9456 & 6.2251 & Positive & 0.6698 & 1.2230 & 0.0177 \\
\hline LMPR04000010 & 2-methylbacteriohopane-32,33,34,35-tetrol & 581.4554 & 6.8783 & Negative & 1.5976 & 1.2967 & 0.0178 \\
\hline HMDB00651 & Decanoylcarnitine & 653.4745 & 8.6022 & Positive & 0.6570 & 1.1854 & 0.0180 \\
\hline HMDB06246 & Tetracosatetraenoic acid (24:4n-6) & 405.2998 & 8.3029 & Negative & 2.3812 & 1.6977 & 0.0186 \\
\hline HMDB04309 & Triterpenoid & 535.2935 & 6.0458 & Positive & 0.6488 & 1.3223 & 0.0189 \\
\hline HMDB11494 & LysoPE(0:0/22:5(4Z,7Z,10Z,13Z,16Z)) & 526.2952 & 5.2339 & Negative & 0.4995 & 1.1323 & 0.0200 \\
\hline HMDB00508 & Ribitol & 455.1960 & 7.4471 & Negative & 1.8272 & 1.0417 & 0.0202 \\
\hline HMDB00027 & Tetrahydrobiopterin & 500.2783 & 5.3003 & Positive & 0.5117 & 1.2442 & 0.0210 \\
\hline HMDB02925 & $8,11,14$-eicosatrienoic acid & 339.2892 & 9.3361 & Positive & 0.6451 & 1.1500 & 0.0220 \\
\hline HMDB29482 & Didymin & 593.1895 & 7.8649 & Negative & 1.9325 & 1.2976 & 0.0224 \\
\hline HMDB09055 & $\operatorname{PE}(18: 1(9 Z) / 16: 0)$ & 794.4496 & 6.2251 & Positive & 0.6046 & 1.2594 & 0.0228 \\
\hline HMDB53219 & $\begin{array}{l}\text { TG(18:3(9Z,12Z,15Z)/20:5(5Z,8Z,11Z,14Z,17Z) } \\
\text { /20:5(5Z,8Z,11Z,14Z,17Z))[iso3] }\end{array}$ & 901.6793 & 7.3508 & Negative & 1.6115 & 1.3523 & 0.0228 \\
\hline HMDB61701 & 2-oleoylglycerophosphocholine & 521.3495 & 5.5826 & Negative & 0.6010 & 1.3357 & 0.0232 \\
\hline
\end{tabular}


TABLE 2 | Continued

\begin{tabular}{|c|c|c|c|c|c|c|c|}
\hline Compound ID & Compound name & $\mathrm{m} / \mathrm{z}$ value & RT (min) & Ion mode & FCvalue ${ }^{b, c}$ & VIPvalue $^{c}$ & pvalue ${ }^{c}$ \\
\hline LMST05010016 & Galpha-glucuronosylhyodeoxycholate & 284.6664 & 6.0595 & Positive & 0.6197 & 1.2900 & 0.0233 \\
\hline HMDB00476 & 3-oxo-4,6-choladienoic acid & 779.4714 & 6.2251 & Positive & 0.5820 & 1.3608 & 0.0234 \\
\hline HMDB00387 & 3-hydroxydodecanoic acid & 293.0903 & 2.1975 & Positive & 0.5924 & 1.4002 & 0.0243 \\
\hline HMDB60579 & Ramiprilat & 425.1488 & 6.4264 & Negative & 1.5224 & 1.3263 & 0.0246 \\
\hline HMDB00949 & Tetrahydrocortisol & 755.4739 & 7.4452 & Positive & 1.7720 & 1.2388 & 0.0263 \\
\hline HMDB03577 & VPGPR Enterostatin & 557.3389 & 6.9452 & Positive & 0.5808 & 1.1159 & 0.0264 \\
\hline HMDB04913 & Ganglioside GD3 (D18:1/16:0) & 804.9525 & 6.2251 & Positive & 0.6007 & 1.3974 & 0.0266 \\
\hline HMDB60508 & Secalciferol & 877.6602 & 6.8783 & Negative & 2.1024 & 1.4062 & 0.0273 \\
\hline HMDB03598 & Retinyl ester & 649.4431 & 6.8783 & Negative & 1.5325 & 1.2533 & 0.0278 \\
\hline HMDB10397 & LysoPC(20:5(5Z,8Z,11Z,14Z,17Z)) & 542.3235 & 5.2315 & Positive & 0.5336 & 1.2340 & 0.0298 \\
\hline HMDB13456 & $\mathrm{PC}(\mathrm{O}-22: 2(13 Z, 16 Z) / 22: 3(10 Z, 13 Z, 16 Z))$ & 878.7119 & 7.3489 & Positive & 2.2710 & 1.0600 & 0.0316 \\
\hline HMDB09210 & PA(18:4(6Z,9Z,12Z,15Z)/22:6(4Z,7Z,10Z,13Z,16Z,19Z)) & 741.4523 & 7.8628 & Positive & 1.9090 & 1.1151 & 0.0320 \\
\hline HMDB00079 & Dihydrothymine & 383.1757 & 6.6995 & Negative & 1.6348 & 1.1711 & 0.0329 \\
\hline HMDB13111 & Ubiquinol-10 & 941.6087 & 6.8764 & Positive & 1.7289 & 1.3385 & 0.0335 \\
\hline HMDB04162 & Galactan & 533.2137 & 8.3010 & Positive & 1.7240 & 1.5959 & 0.0340 \\
\hline HMDB07046 & $\mathrm{DG}(14: 1(9 Z) / 18: 3(6 Z, 9 Z, 12 Z) / 0: 0)$ & 605.1058 & 6.6995 & Negative & 2.2738 & 1.3274 & 0.0341 \\
\hline HMDB02596 & Deoxycholic acid 3-glucuronide & 607.2992 & 6.9452 & Positive & 0.6623 & 1.0737 & 0.0356 \\
\hline HMDB13622 & Nonadeca-10(Z)-enoic acid & 295.2644 & 7.6170 & Negative & 1.7845 & 1.3472 & 0.0357 \\
\hline HMDB01999 & Eicosapentaenoic acid & 301.2182 & 8.5553 & Negative & 0.4157 & 1.0496 & 0.0427 \\
\hline HMDB04863 & Ganglioside GM1 (d18:1/24:0) & 825.0025 & 6.9452 & Positive & 0.5165 & 1.2551 & 0.0442 \\
\hline HMDB11489 & LysoPE(0:0/20:5(5Z,8Z,11Z,14Z,17Z)) & 498.2638 & 5.3026 & Negative & 0.5686 & 1.2098 & 0.0463 \\
\hline HMDB11891 & Ganglioside GM1 (d18:1/18:1(11Z)) & 513.2805 & 5.1514 & Negative & 2.6454 & 1.0136 & 0.0468 \\
\hline HMDB01138 & $\mathrm{N}$-acetylglutamic acid & 379.1386 & 6.6976 & Positive & 1.6936 & 1.2277 & 0.0473 \\
\hline HMDB32033 & $2,4,12$-octadecatrienoic acid isobutylamide & 378.2729 & 9.0679 & Positive & 0.6475 & 1.0131 & 0.0474 \\
\hline HMDB00962 & Lipoamide & 586.3166 & 5.0825 & Negative & 0.4844 & 1.1850 & 0.0479 \\
\hline HMDB00319 & 18-hydroxycorticosterone & 395.2425 & 8.3010 & Positive & 1.6211 & 1.6175 & 0.0484 \\
\hline HMDB07951 & PC(15:0/20:5(5Z,8Z,11Z,14Z,17Z)) & 810.5286 & 8.4060 & Negative & 0.4467 & 1.0508 & 0.0489 \\
\hline HMDB32002 & Dehydrotomatine & 352.1781 & 6.6976 & Positive & 1.5554 & 1.1068 & 0.0492 \\
\hline
\end{tabular}

${ }^{a}$ Compound ID was mainly exhibited based on the Human Metabolome Database (www.hmdb.ca), and the others based on National Center for Biotechnology Information (www.ncbi. nIm.nih.gov/pccompound/) or LIPID MAPS (www.lipidmaps.org).

${ }^{b} F C$ value was calculated as the ratio of the average mass response (area) between the two groups ( $F C$ value $\left.=P D D / P D\right)$. Thus, FC values $>1$ indicate significantly higher levels in the $P D D$ group relative to the $P D$ group while $F C$ values $<1$ indicate significantly lower levels in the PDD group.

${ }^{c}$ Only metabolites with $F C$ values greater than \pm 1.5 , VIP values greater than 1.0 and $p$ values less than 0.05 were deemed statistically significant.

$P D$, Parkinson's disease; RT, retention time; FC, fold change; VIP, variable influence on projection; PDD, Parkinson's disease related depression.

1-(alpha-Methyl-4-(2-methylpropyl)benzeneacetate)-betaD-Glucopyranuronic acid). Those differentially expressed metabolites were primarily located in the extracellular space
(41\%), membrane (38\%), cytoplasm (11\%), mitochondria (4\%), endoplasmic reticulum (1\%), and nucleus (1\%) (Figure 2D). 


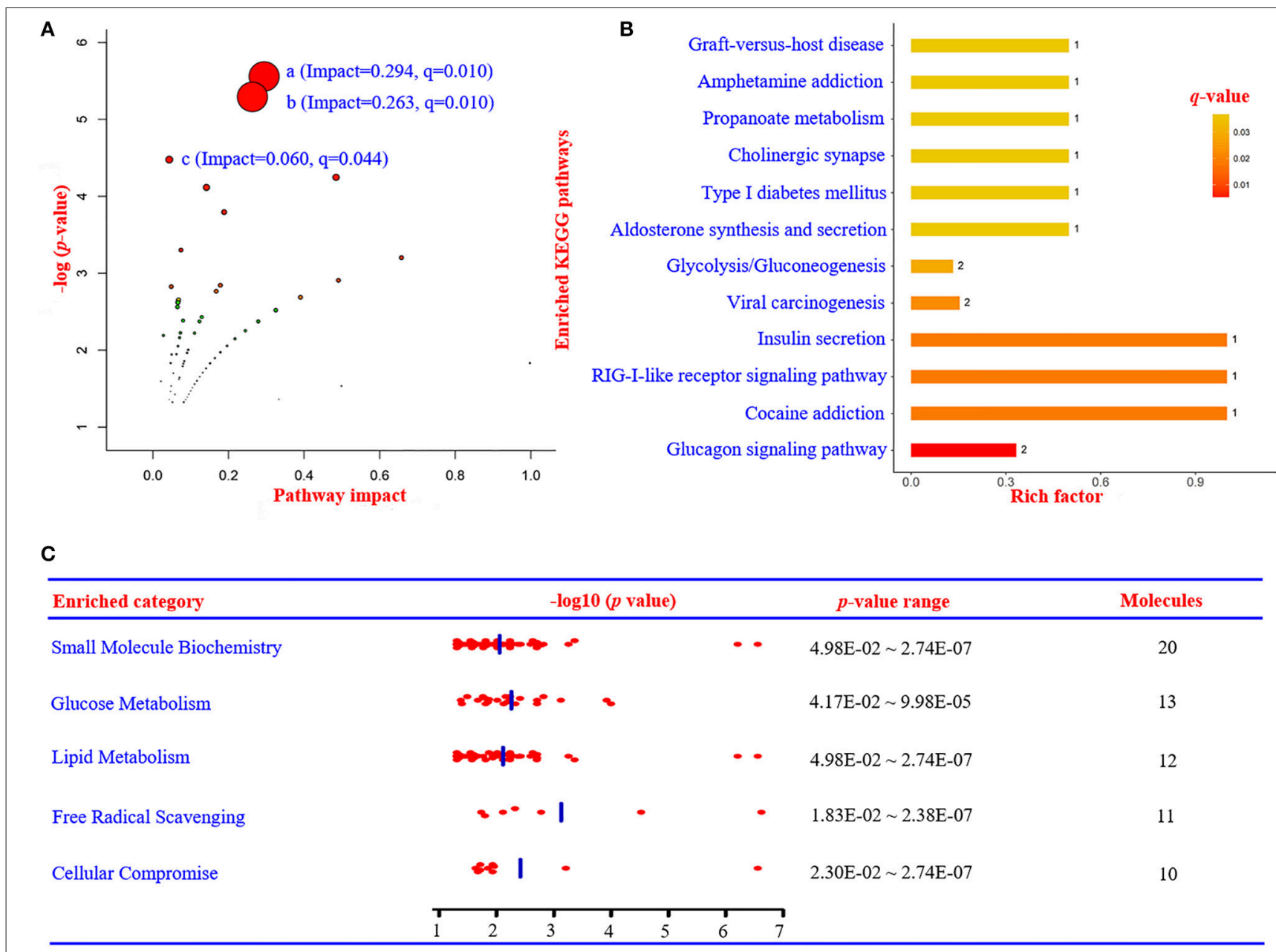

FIGURE 3 | Summary of metabolic pathways based on metabolomics, proteomics, and integrated analysis. (A) Metabolic pathway analysis based on metabolomics data using IMPaLA. (a) Alpha-linolenic acid and linoleic acid metabolism (impact $=0.294, q=0.010$ ). (b) Incretin synthesis, secretion, and inactivation (impact $=0.263, q=0.010$ ). (c) Signal transduction (impact $=0.060, q=0.044$ ). (B) Enriched KEGG pathways based on proteomics data. (C) The most significant enriched function annotations analyzed by Ingenuity Pathways Analysis, based on the differentially expressed metabolites and proteins between patients with PDD and PD. KEGG, Kyoto Encyclopedia of Genes and Genomes; PDD, Parkinson's disease-related depression; PD, Parkinson's disease.

The related metabolic pathways are shown in Figure 3A and Supplementary Table 2, with three most significant pathways: (1) alpha-linolenic acid and linoleic acid metabolism (pathway impact $=0.294, q$-value $=0.0102$ ); (2) incretin synthesis, secretion, and inactivation (pathway impact $=0.263$, $q$-value $=0.0102$ ); and (3) signal transduction (pathway impact $=0.060, q$-value $=0.0443)$.

\section{Proteomics Analysis}

The clinical characteristics of patients included in the TMT-based proteomics analysis are shown in Supplementary Table 3 and were also comparable with the total patient group. Identification and evaluation of the quantitative proteomics results are shown in Figure 4. A total of 912 unique proteins were identified and protein ratio distributions between the two groups are shown in Figure 5. Only 17 differentially expressed proteins were selected for further analysis (Table 3).
There were 73, 14, 23 significant GO terms related to the differentially expressed proteins associated with biological process, cellular component, and molecular function, respectively. The top 20 GO terms of GO enrichment analysis are shown in Figure 6 and Supplementary Table 4. Several top ranking GO terms from biological processes were associated with glucose metabolism, including single-organism carbohydrate catabolic process, carbohydrate catabolic process, monosaccharide catabolic process, and hexose catabolic process. The top ranking enriched terms that related to molecular function were glycerone kinase activity, transcription factor activity, ligand-activated RNA polymerase II transcription factor binding, T cell receptor binding, and triokinase activity. The top ranking enriched terms that related to cellular components were anchoring collagen complex and Golgi medial cisterna.

Differentially expressed proteins were also mapped to KEGG pathways and enriched in 12 specific pathways (Figure 3B 

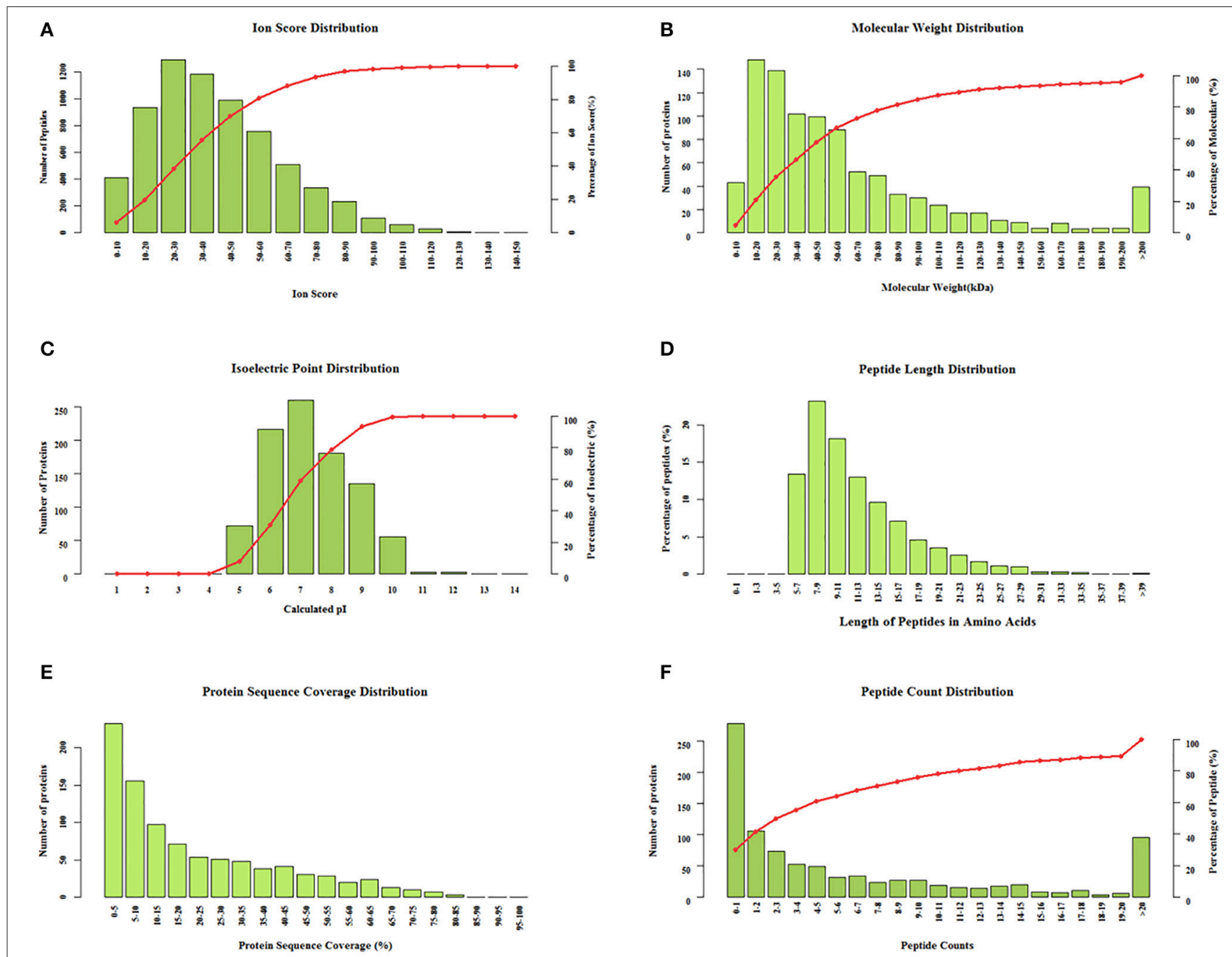

FIGURE 4 | Identification and evaluation of the quantitative results from the tandem mass tag-based proteomics analysis. Protein distributions according to (A) ion score, (B) molecular weight, (C) isoelectric point, (D) peptide length, (E) protein sequence coverage, and (F) peptide count.

and Supplementary Table 5) with $q$-values $<0.05$. There were six statistically overrepresented pathways involved in glucose metabolism, including glucagon signaling pathway, insulin secretion, glycolysis/gluconeogenesis, aldosterone synthesis and secretion, type I diabetes mellitus, and propanoate metabolism. All together, these findings further point to altered glucose metabolism in the PDD group.

\section{Integrated Analysis}

According to IPA analysis, these differentially expressed metabolites and proteins significantly relate to many molecular or cellular functions (Supplementary Table 6), further enriched in the following five categories: small molecule biochemistry, glucose metabolism, lipid metabolism, free radical scavenging, and cellular compromise (Figure 3C). The main metabolite-protein integrated network was "cellular compromise, lipid metabolism, and small molecule biochemistry" with a $p$ score of 80 and a total of 27 metabolites and 9 proteins involved in the network (Figure 7).

\section{ELISA Tests}

According to the ELISA results (Table 4), the plasma levels of PTPRZ1 and NOTCH2 were significantly increased in patients with PDD, whereas the plasma levels of HLA-A, LPA, LDHA, and GAPDH were significantly decreased (Figure 8A). Correlation analyses revealed significant positive associations of PTPRZ1 and NOTCH2 and negative associations of HLAA, LPA, LDHA, and GAPDH with HAMD scores (Figure 8B). PTPRZ1 also exhibited negative associations with HLA-A, LDHA, and GAPDH, whereas LPA was positively associated with LDHA. Receiver operating characteristic (ROC) curves were further analyzed and diagnostic efficacies were compared. Among them, the AUC value of NOTCH2 was significantly higher than the others. It suggested the plasma NOTCH2 level to be the best potential biomarker for patients with PDD 
(Figure 8C), with a sensitivity and specificity of 95.65 and $81.58 \%$, respectively, and an optimal cut-off point of $0.91 \mathrm{ng} / \mathrm{ml}$ (Figure 8D).

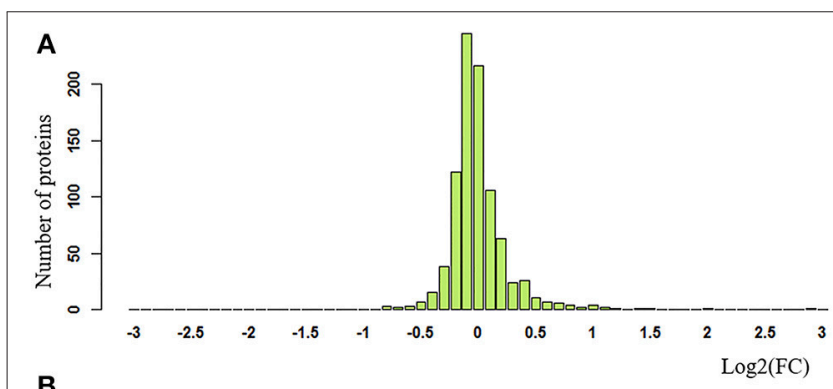

$\mathbf{B}$

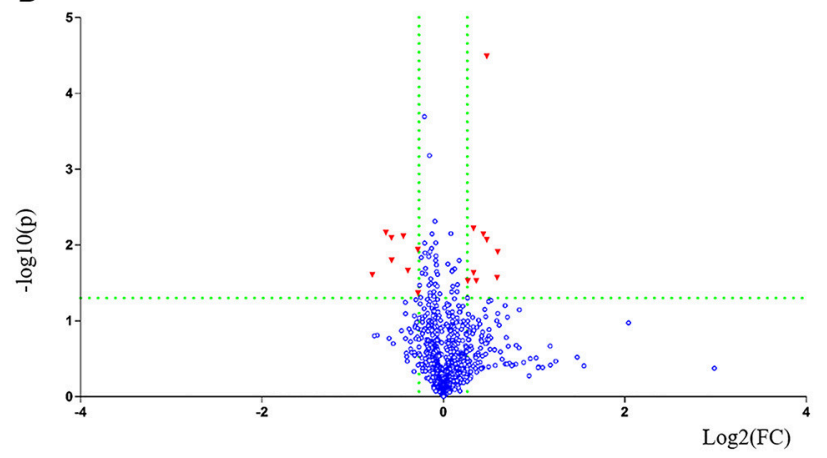

FIGURE $\mathbf{5}$ | Statistical comparison and exhibition of proteins between patients with PDD and PD in proteomics analysis. (A) Protein ratio distribution and (B) volcano plot. FC value was calculated as the ratio of the average mass response (area) between the two groups ( $F C$ value $=\mathrm{PDD} / \mathrm{PD})$. $P$-value was calculated using Mann-Whitney U-test. PDD, Parkinson's disease-related depression; PD, Parkinson's disease; FC, fold change.

\section{DISCUSSION}

Research bridging systems biology and clinical characteristics is useful to explore the underlying pathogenesis and to identify high quality clinical biomarkers (Mostafa et al., 2016). Here, we have performed an integrated metabolomics and proteomics analysis of PDD for the first time and have further identified potential blood biomarkers for depression in patients with PD.

No significant differences were found in most of the clinical characteristics, including age and gender, suggesting that our comparisons were reasonable. Hypertension is reportedly more prevalent in patients with PD (Hsu et al., 2015) and patients with depression (Yu et al., 2017), whereas patients with PDD in our research suffered less hypertension. According to a research from South Korea (Park et al., 2016), the incidence of hypertension is not significantly different between patients with PD with and without depression. This inconsistency is probably because the disease durations of patients with PD from South Korea were significantly shorter than those in our study $(2.0 \pm 1.0$ vs. $6.0 \pm 5.1$ years). Orthostatic hypotension is reported to be associated with depressive symptoms in patients with PD, and that patients with PDD have attenuated cardiovagal dysfunction as observed during ambulation (Park et al., 2016). Decreased blood pressure in patients with PDD probably appears as the disease progresses. Serum lipid levels (triglyceride, Yu et al., 2017, LDL-C, Persons and Fiedorowicz, 2016, and high-density lipoprotein cholesterol, Ong et al., 2016) are also reportedly associated with depressive symptoms and suicidality (Oh and Kim, 2017). Here, we have demonstrated significantly lower serum LDL-C and Apo-B levels in patients with PDD for the first time, indicating that altered lipid metabolism is also associated with PDD. However, the incidence of hypercholesterolemia

TABLE 3 | Key differentially expressed proteins identified by tandem mass tag-based proteomics analysis between patients with PD with and without depression.

\begin{tabular}{|c|c|c|c|c|}
\hline UniProt ID & Protein name (GeneName) & MW [kDa] & FC value ${ }^{a}$ & $p$-value ${ }^{b}$ \\
\hline B3KY04 & Potassium channel tetramerization domain containing 12 (KCTD12) & 35.71 & 1.392 & 0.000 \\
\hline V9HW68 & Epididymis luminal protein 214 (HEL-214) & 51.68 & 1.258 & 0.006 \\
\hline Q68CJ9 & Cyclic AMP-responsive element-binding protein 3-like protein 3 (CREB3L3) & 49.05 & 1.357 & 0.007 \\
\hline E9PNH1 & Neutral alpha-glucosidase AB (GANAB) & 13.15 & 1.392 & 0.009 \\
\hline Q53HT9 & Complement component $1, r$ subcomponent variant & 80.19 & 1.514 & 0.012 \\
\hline P23471 & Receptor-type tyrosine-protein phosphatase zeta (PTPRZ1) & 254.43 & 1.259 & 0.023 \\
\hline A0A075B6R9 & Protein IGKV2D-24 (IGKV2D-24) & 13.07 & 1.506 & 0.027 \\
\hline A0A125U0U7 & MS-C1 heavy chain variable region & 13.09 & 1.286 & 0.030 \\
\hline Q04721 & Neurogenic locus notch homolog protein 2 (NOTCH2) & 265.23 & 1.204 & 0.030 \\
\hline P00338 & L-lactate dehydrogenase A chain (LDHA) & 36.67 & 0.644 & 0.007 \\
\hline U5YKD2 & MHC class I antigen (HLA-A) & 37.91 & 0.736 & 0.008 \\
\hline Q1HP67 & Lipoprotein (LPA) & 226.37 & 0.673 & 0.008 \\
\hline Q8NCM2 & Potassium voltage-gated channel subfamily $\mathrm{H}$ member 5 (KCNH5) & 111.81 & 0.822 & 0.012 \\
\hline E7EUT5 & Glyceraldehyde-3-phosphate dehydrogenase (GAPDH) & 27.85 & 0.673 & 0.016 \\
\hline Q5NV68 & V4-1 protein (V4-1) & 11.23 & 0.762 & 0.022 \\
\hline I3L252 & Triokinase/FMN cyclase (TKFC) & 22.94 & 0.581 & 0.025 \\
\hline D6RGG3 & Collagen alpha-1(XII) chain (COL12A1) & 333.00 & 0.824 & 0.043 \\
\hline
\end{tabular}

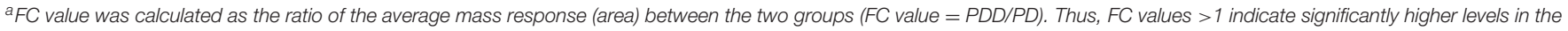
$P D D$ group relative to the $P D$ group while FC values $<1$ indicate significantly lower levels in the PDD group.

${ }^{b}$ Only proteins with $F C$ values greater than \pm 1.2 and $p$ values less than 0.05 were deemed statistically significant.

PD, Parkinson's disease; MW, molecular weight; FC, fold change; PDD, Parkinson's disease related depression. 


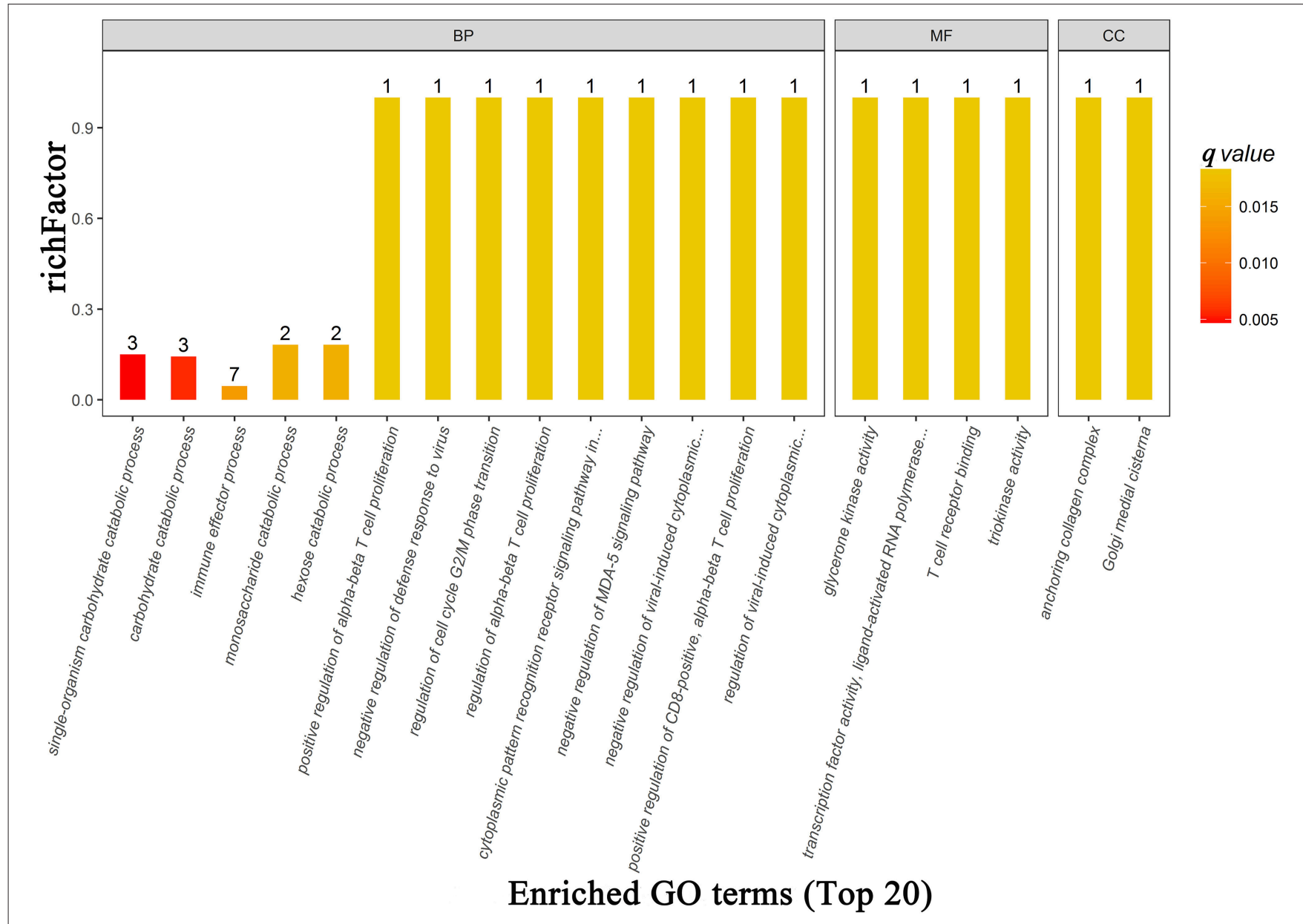

FIGURE 6 | The top 20 most enriched GO terms based on proteomics analysis between patients with PDD and PD. BP, biological process; MF, molecular function; CC, cellular component; GO, gene ontology; PDD, Parkinson's disease-related depression; PD, Parkinson's disease.

in patients with PDD exhibited no significant difference, probably because the altered lipid metabolism was well below that needed for a clinical diagnosis of hypercholesterolemia. Glucose metabolism is also reportedly associated with depressive symptoms in major depressive disorder (Koponen et al., 2015), post-stroke depression (Zhang et al., 2017b), and prenatal depressive symptoms (Huang et al., 2015). Patients with diabetes mellitus have a greater incidence of depressed mood compared to patients without diabetes mellitus (Levinger et al., 2012). Yet, the serum levels of blood glucose and $\mathrm{HbA1C}$ in our study showed no significant differences between patients with PDD and PD. In addition, the incidence of diabetes mellitus did not differ between patients with PD with and without depression (Icks et al., 2013). This is probably because the depressive systems are mainly correlated with impaired insulin sensitivity ( $\mathrm{Li}$ et al., 2016) or low insulin secretion (Akbaraly et al., 2013), and the risk of depression gradually increases along with the deterioration of glucose metabolism (Chen et al., 2016).

Metabolomics analyses have already been performed in patients with PD (Trezzi et al., 2017) and patients with depression (Liu et al., 2015). Several metabolic pathways, including catecholamine metabolism, caffeine and xanthine pathways, ornithine pathway, and redox homoeostasis, are reportedly altered in patients with PD (Hatano et al., 2016), while depressive symptoms are associated with lipid metabolism, carnitine metabolism, amino acid metabolism, and bile acid metabolism (Liu et al., 2015). Nevertheless, this metabolomics analysis was the first to be performed in patients with PDD compared with patients with PD and significant metabolite changes were identified. Most of the differentially expressed metabolites were categorized into lipids and lipid-like molecules. Four carbohydrates had also changed. The involvement of lipid metabolism in PDD is consistent with the above clinical characteristics (lower LDL-C and Apo-B level) and can further be confirmed by the metabolic pathway analysis. The most significant and important pathway was "alpha linolenic acid and linoleic acid metabolism," participating in the metabolism of essential polyunsaturated fatty acids. This metabolic pathway is reportedly associated with the occurrence (Lucas et al., 2011) and treatment (Kanno et al., 2015) of depression, and the weak positive association between the 


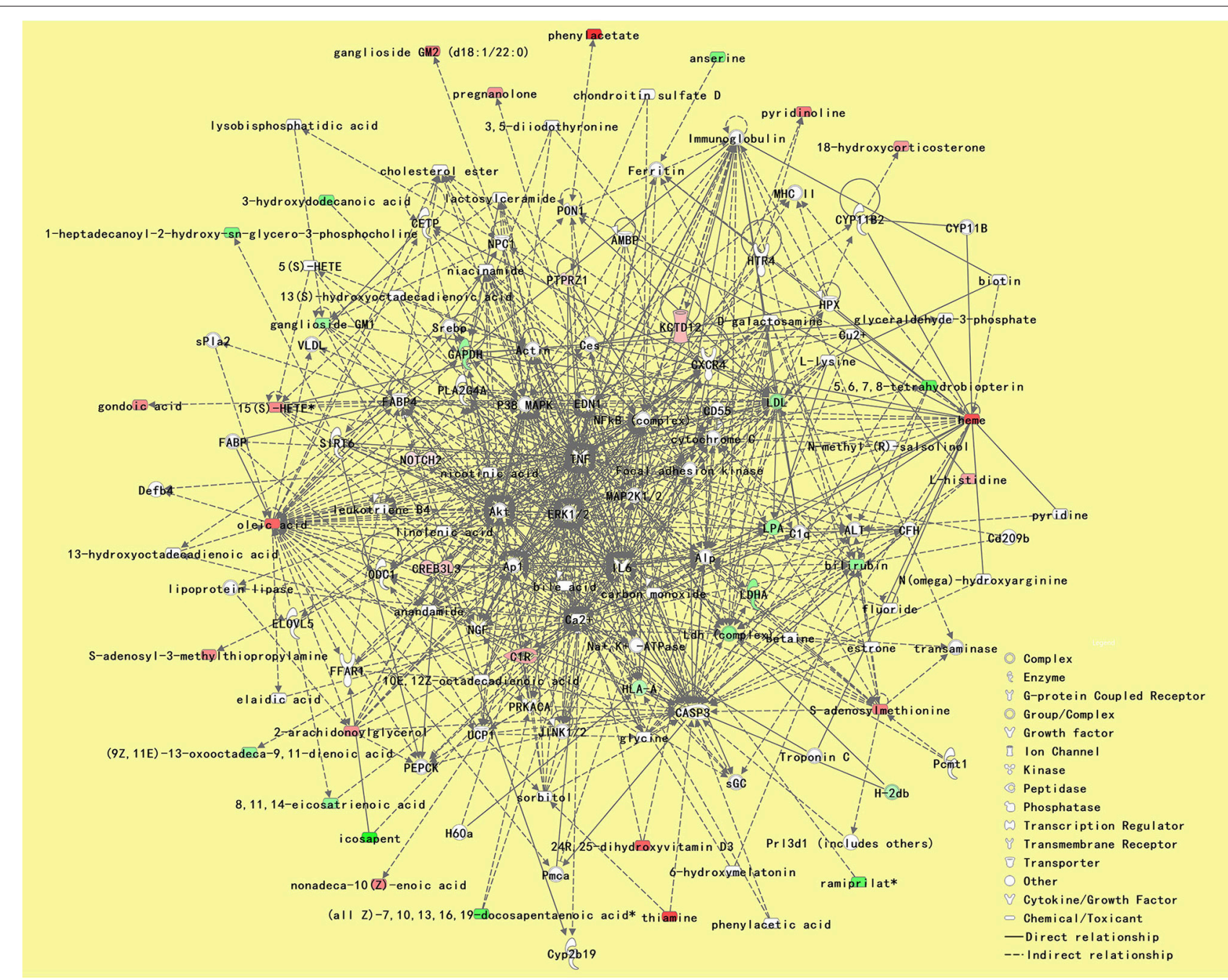

FIGURE 7 | The most significant metabolite-protein integrated network between patients with PDD and PD. The network was "cellular compromise, lipid metabolism, and small molecule biochemistry" with a pscore of 80, and a total of 27 metabolites and 9 proteins involved in the network. Upregulated metabolite symbols are in red, while green symbols indicate downregulated metabolites between patients with PDD and the other patients with PD. PDD, Parkinson's disease-related depression; PD, Parkinson's disease.

intake of polyunsaturated fatty acid and PD risk needs further investigation (Dong et al., 2014). The second most significantly altered metabolic pathway was "incretin synthesis, secretion, and inactivation," which is related to glucose metabolism. The decreased secretion and sensitivity level of insulin, another common hypoglycemic hormone, appears to be a risk factor for depression (Akbaraly et al., 2013; Li et al., 2016). However, this is the first report of the synthesis, secretion, and inactivation of incretin involved in patients with PDD, and the relationship between incretin and depressive symptoms in patients with PD needs further clarification. The last significant pathway was "signal transduction," by which a chemical or physical signal is transmitted through a cell, ultimately resulting in a cellular response (Hynes et al., 2013). The realization of altered lipid and glucose metabolism is also through such a process.
A previous serum proteomics analysis revealed that oxidative stress, mitochondrial dysfunction, abnormal protein aggregation, and inflammation may be associated with PD (Zhang et al., 2012), while patients with depression may have altered lipid metabolism and immunoregulation (Xu et al., 2012). In this TMT-based proteomics analysis, 17 differentially expressed proteins were identified for further analysis. According to the GO enrichment analysis, four of the top five ranking GO terms from biological processes were associated with glucose metabolism, including single-organism carbohydrate catabolic process, carbohydrate catabolic process, monosaccharide catabolic process, and hexose catabolic process. Enriched KEGG pathway analysis was also performed and 12 statistically overrepresented pathways were mapped, among which six pathways were involved in glucose metabolism, including glucagon signaling pathway, insulin secretion, glycolysis/gluconeogenesis, aldosterone synthesis and 


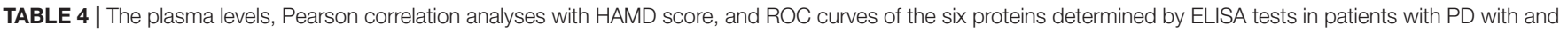
without depression.

\begin{tabular}{|c|c|c|c|c|c|c|c|c|c|}
\hline \multirow[t]{2}{*}{ Variable } & \multicolumn{3}{|c|}{ Mean \pm SEM (ng/ml) } & \multicolumn{2}{|c|}{ Correlation analysis } & \multicolumn{4}{|c|}{ ROC curve } \\
\hline & PD (76) & PDD (23) & $p$-value & r value & $p$ value & AUC $(95 \% \mathrm{Cl})$ & $\begin{array}{l}\text { Cut-off point } \\
\text { (ng/ml) }\end{array}$ & $\begin{array}{l}\text { Sensitivity } \\
\text { value (\%) }\end{array}$ & $\begin{array}{c}\text { Specificity } \\
\text { value (\%) }\end{array}$ \\
\hline PTPRZ1 & $1.88 \pm 0.17$ & $2.68 \pm 0.28$ & 0.000 & 0.417 & 0.000 & $0.758(0.662-0.838)$ & 1.564 & 86.96 & 64.47 \\
\hline HLA-A & $0.41 \pm 0.03$ & $0.29 \pm 0.01$ & 0.013 & -0.272 & 0.006 & $0.672(0.570-0.763)$ & 18.738 & 100.0 & 39.47 \\
\hline NOTCH2 & $0.94 \pm 0.19$ & $1.56 \pm 0.24$ & 0.000 & 0.335 & 0.001 & 0.910 (0.835-0.958) & 0.857 & 95.65 & 81.58 \\
\hline LPA & $209.56 \pm 10.76$ & $151.90 \pm 11.14$ & 0.009 & -0.354 & 0.000 & $0.681(0.579-0.771)$ & 216.827 & 95.65 & 44.74 \\
\hline LDHA & $10.83 \pm 1.62$ & $5.05 \pm 1.07$ & 0.003 & -0.251 & 0.012 & $0.707(0.607-0.794)$ & 4.542 & 78.26 & 63.16 \\
\hline GAPDH & $6.29 \pm 0.33$ & $4.77 \pm 0.39$ & 0.028 & -0.366 & 0.000 & $0.652(0.550-0.745)$ & 6.203 & 86.96 & 40.79 \\
\hline
\end{tabular}

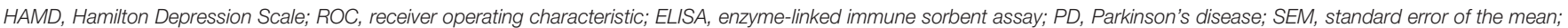

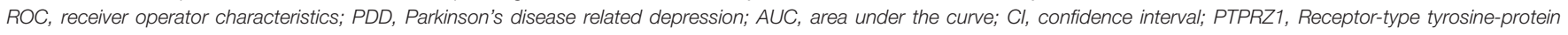

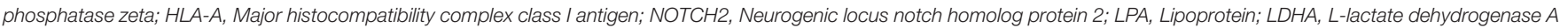
chain; GAPDH, Glyceraldehyde-3-phosphate dehydrogenase.

secretion, type I diabetes mellitus, and propanoate metabolism. Those findings at the protein level firmly support the results of the metabolomics analysis. Although the involvement of lipid metabolism was not confirmed at the protein level by pathway analysis, lysophosphatidic acid (LPA), a common protein related to lipid metabolism was significantly increased in patients with PDD based on proteomics analysis. In other words, both glucose and lipid participate in the energy metabolism of a biological system. It is just the alteration of glucose metabolism at the protein level that induces disturbance of lipid and glucose metabolism at the metabolite level.

Metabolic pathways involve both metabolites and proteins, and integrated analysis of both metabolomics and proteomics data will thus help us interpret the above inconsistence (Zhang et al., 2017a). When incorporating all the differentially expressed metabolites and proteins onto indole-3-propionic acid (IPA), those differentially expressed molecules predominantly participated in small molecule biochemistry, glucose metabolism, lipid metabolism, free radical scavenging, and cellular compromise. Small molecule biochemistry is the metabolic processes of many small molecules, including glucose and lipid metabolism, consistent with the above results of the clinical characteristics, metabolomics, and proteomics. Oxidative stress is one of the mechanisms of cellular damage and can also induce depressive symptoms according to previous research (Pan et al., 2014; Bouvier et al., 2016). The enriched category of free radical scavenging in this study includes the production and activation of reactive oxygen species, while cellular compromise includes cytotoxicity and cellular injury (Supplementary Table 6). Those results demonstrate that altered lipid and glucose metabolism induces depressive symptoms in patients with $\mathrm{PD}$, possibly also through oxidative stress and the resulting cellular injury. The most significant integrated network in this study was "cellular compromise, lipid metabolism, and small molecule biochemistry," with a total of 27 metabolites and 9 proteins involved. Those molecules in the network were recognized as the most relevant ones with depressive systems in patients with PD (Dong et al., 2017b). We further performed ELISA tests for six differentially expressed proteins to identify some clinical biomarkers as an integral part of clinical researches (Mostafa et al., 2016).

The expression differences of those proteins were confirmed. Correlation analyses revealed significant positive associations of PTPRZ1 and NOTCH2 and negative associations of HLAA, LPA, LDHA, and GAPDH, with HAMD scores. Significant correlations were also found between these proteins. PTPRZ1 exhibited negative associations with HLA-A, LDHA, and GAPDH, whereas LPA was positively associated with LDHA. For these proteins ROC curves were performed, and the comparison of diagnostic efficacies identified the plasma NOTCH2 level to be the best potential blood biomarker for PDD (with a significantly higher AUC value than the others). NOTCH2 can be expressed in various cell types from the hematolymphoid compartment and has specific roles in differentiation and function of many immune cells (Sakata-Yanagimoto and Chiba, 2012). Genetic variants of NOTCH2 reportedly can also increase susceptibility to diabetes mellitus (Pan et al., 2013). Hence, the increased plasma NOTCH2 level in patients with PD induces depressive symptoms probably also through glucose metabolism.

There were several limitations to this study. First, the number of patients included was relatively small, and all the patients were from a Chinese Han population. The results require further confirmation, especially in another ethnicity. Second, we have only determined six differentially expressed proteins according to the metabolite-protein integrated network. More antibodies and ELISA kits should be developed to validate the alteration of the other proteins. We hope to continue the research about PDD and overcome these limitations in the future.

\section{CONCLUSIONS}

The integrated metabolomics and proteomics analysis from this study reveals that depressive symptoms in patients with PD are predominantly associated with lipid and glucose metabolism. Plasma NOTCH2 level may be a good blood biomarker for detecting patients of PD with depression. 
A

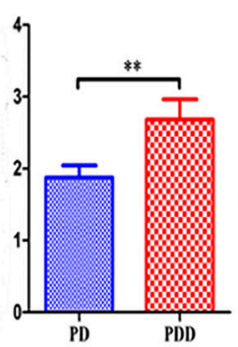

PTPRZ1 (ng/ml)

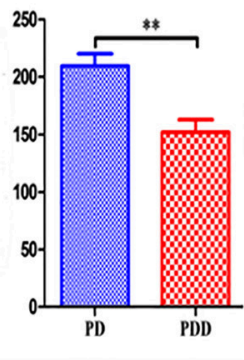

LPA (ng/ml)

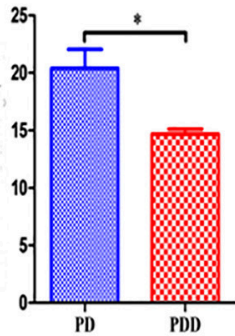

HLA-A (ng/ml)

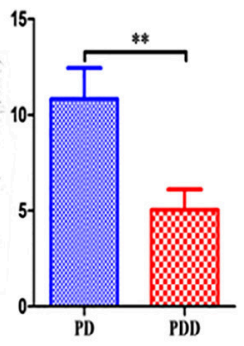

LDHA (ng/ml)

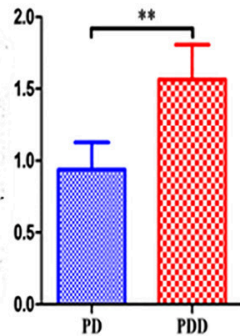

NOTCH2 (ng/ml)

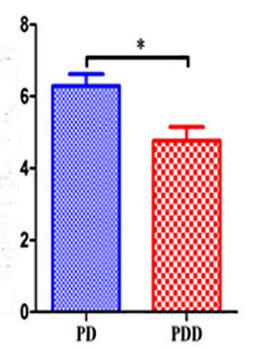

GAPDH (ng/ml)
B

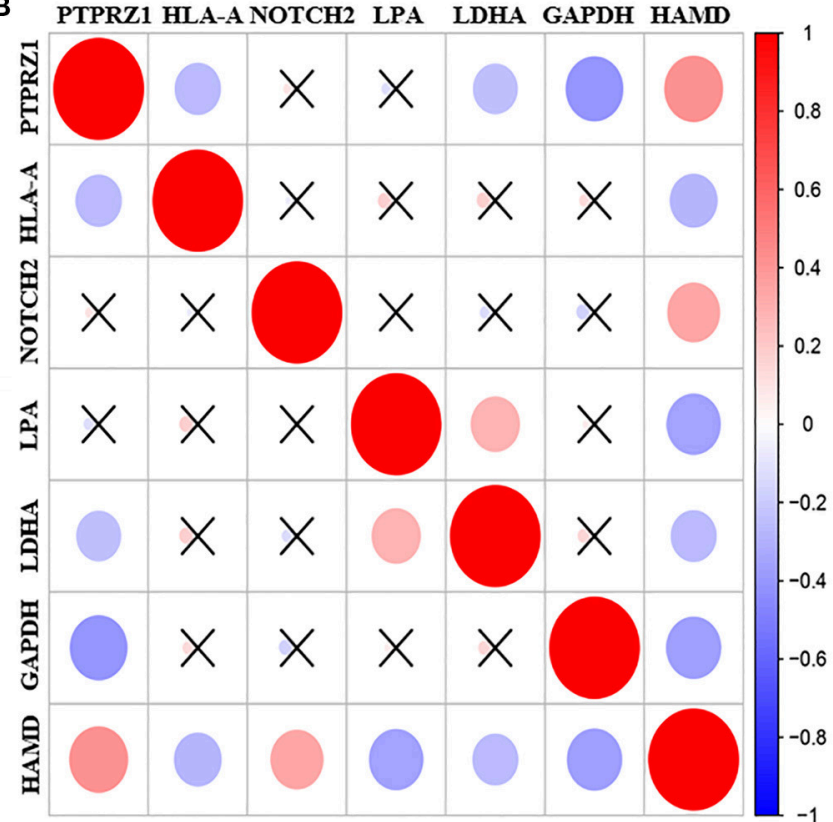

C

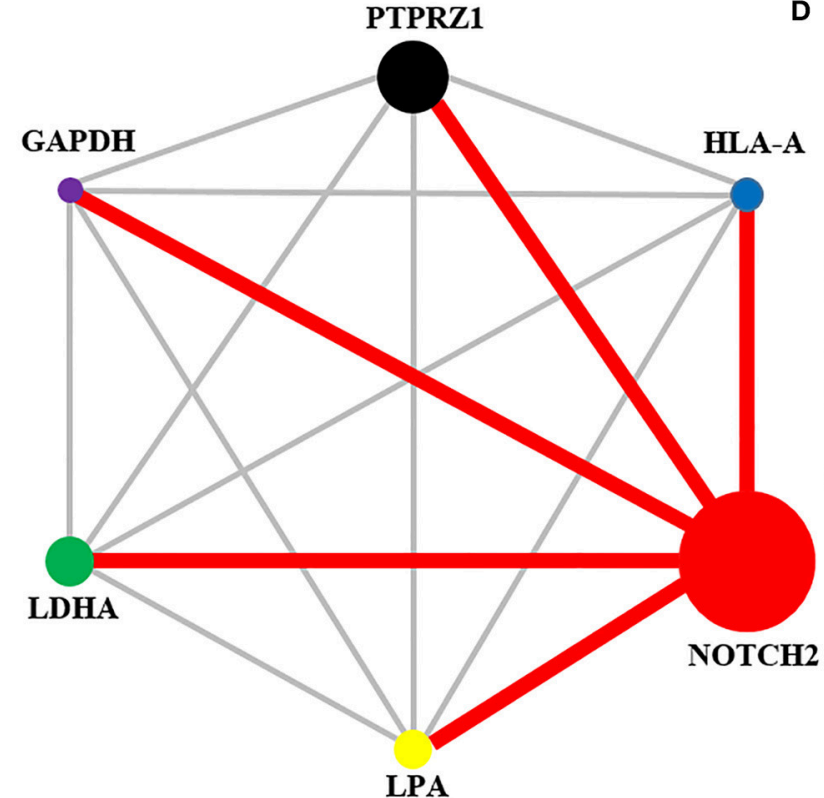

D

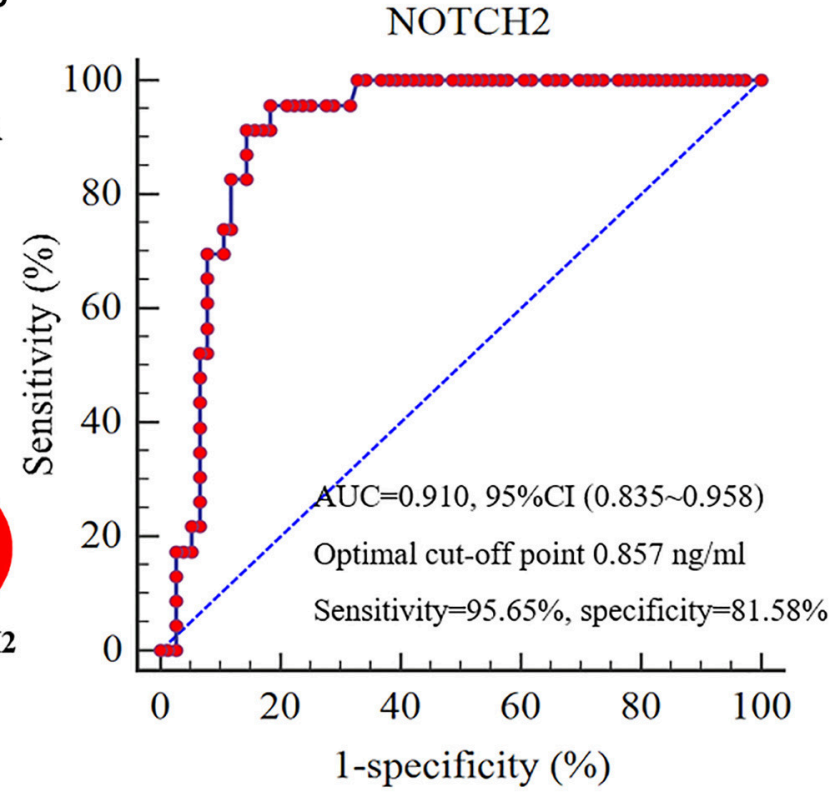

FIGURE 8 | NOTCH2 may be a good potential biomarker for patients with PDD based on ROC curves. (A) Comparisons of the plasma levels of six proteins determined by ELISA tests between patients with PDD and the other patients with PD. The plasma levels of PTPRZ1 andNOTCH2 significantly increased in patients with PDD, whereas the plasma levels of HLA-A, LPA, LDHA, and GAPDH significantly decreased compared with the patients with PD. ${ }^{*} p<0.05$ and ${ }^{* *} p<0.01$ compared with the patients with PD. (B) Correlation analyses between the plasma levels of six proteins and HAMD score in all patients with PD. The vertical bar on the right represents correlation coefficient value and " $x$ " indicates no significant correlation can be found between them. The plot indicates positive associations of PTPRZ1 and NOTCH2, and negative associations of HLA-A, LPA, LDHA, and GAPDH, with HAMD scores. PTPRZ1 also exhibited negative associations with HLA-A, LDHA, and GAPDH, whereas LPA was positively associated with LDHA. (C) Comparison network of the diagnostic efficacies for patients with PDD. The circle area represents the AUC value of relevant proteins, and the red line indicates a statistically significant difference of the diagnostic efficacies between the two proteins. The AUC value of NOTCH2 was significantly higher than the others. (D) ROC curve of the plasma levels of NOTCH2 for detecting patients with PDD. PDD, Parkinson's disease-related depression; PD, Parkinson's disease; HAMD, Hamilton Depression Scale; ROC, receiver operating characteristic; AUC, area under the curve; CI, confidence interval. 


\section{DATA AVAILABILITY STATEMENTS}

The raw data supporting the conclusions of this manuscript will be made available by the authors, without undue reservation, to any qualified researcher.

\section{AUTHOR CONTRIBUTIONS}

$\mathrm{M}-\mathrm{XD}$ and Y-DW designed this study. M-XD, XF, X-MX, YL, S-YJ, and LH assessed clinical data. M-XD, BL, and WC performed experiments. M-XD wrote the manuscript. Y-DW revised it.

\section{FUNDING}

This work was supported by Chongqing Health and Family Planning commission (No. 2017MSXM023), Chongqing Science

\section{REFERENCES}

Aarsland, D., Zaccai, J., and Brayne, C. (2005). A systematic review of prevalence studies of dementia in Parkinson's disease. Mov. Disord 20, 1255-1263. doi: $10.1002 / \mathrm{mds} .20527$

Adams-Carr, K. L., Bestwick, J. P., Shribman, S., Lees, A., Schrag, A., and Noyce, A. J. (2016). Constipation preceding Parkinson's disease: a systematic review and meta-analysis. J. Neurol. Neurosurg. Psychiatry 87, 710-716. doi: 10.1136/jnnp-2015-311680

Akbaraly, T. N., Kumari, M., Head, J., Ritchie, K., Ancelin, M. L., Tabak, A. G., et al. (2013). Glycemia, insulin resistance, insulin secretion, and risk of depressive symptoms in middle age. Diab. Care 36, 928-934. doi: 10.2337/dc 12-0239

Altelaar, A. F., Munoz, J., and Heck, A. J. (2013). Next-generation proteomics: towards an integrative view of proteome dynamics. Nat. Rev. Genet. 14, 35-48. doi: $10.1038 / \operatorname{nrg} 3356$

Berardelli, A., Wenning, G. K., Antonini, A., Berg, D., Bloem, B. R., Bonifati, V., et al. (2013). EFNS/MDS-ES/ENS [corrected] recommendations for the diagnosis of Parkinson's disease. Eur. J. Neurol. 20, 16-34. doi: 10.1111/ene.12022

Berger, B., Peng, J., and Singh, M. (2013). Computational solutions for omics data. Nat. Rev. Genet. 14, 333-346. doi: 10.1038/ $\operatorname{nrg} 3433$

Bouvier, E., Brouillard, F., Molet, J., Claverie, D., Cabungcal, J. H., Cresto, N., et al. (2016). Nrf2-dependent persistent oxidative stress results in stress-induced vulnerability to depression. Mol. Psychiatry 22, 1701-1713. doi: $10.1038 / \mathrm{mp} .2016 .144$

Broen, M. P., Narayen, N. E., Kuijf, M. L., Dissanayaka, N. N., and Leentjens, A. F. (2016). Prevalence of anxiety in Parkinson's disease: a systematic review and meta-analysis. Mov. Disord. 31, 1125-1133. doi: 10.1002/mds. 26643

Campbell, K., Xia, J., and Nielsen, J. (2017). The impact of systems biology on bioprocessing. Trends Biotechnol. 35, 1156-1168. doi: 10.1016/j.tibtech.2017.08.011

Chen, S., Zhang, Q., Dai, G., Hu, J., Zhu, C., Su, L., et al. (2016). Association of depression with pre-diabetes, undiagnosed diabetes, and previously diagnosed diabetes: a meta-analysis. Endocrine 53, 35-46. doi: 10.1007/s12020-0160869-x

Dayon, L., Nunez Galindo, A., Corthesy, J., Cominetti, O., and Kussmann, M. (2014). Comprehensive and Scalable Highly Automated MSBased Proteomic Workflow for Clinical Biomarker Discovery in Human Plasma. J. Proteome Res. 13, 3837-3845. doi: 10.1021/pr50 $0635 f$ and technology commission (No. cstc2016jcy jA0423) and National Key Clinical Specialties Construction Program of China.

\section{ACKNOWLEDGMENTS}

We thank Shi-Sheng Wang and his Wu Kong platform for technical support. Wendy Brooks, PhD, provided English language editing services for a draft of this manuscript.

\section{SUPPLEMENTARY MATERIAL}

The Supplementary Material for this article can be found online at: https://www.frontiersin.org/articles/10.3389/fnmol. 2018.00257/full\#supplementary-material

Dissanayaka, N. N., Sellbach, A., Matheson, S., Marsh, R., Silburn, P. A O'sullivan, J. D., et al. (2007). Validity of Hamilton Depression Inventory in Parkinson's disease. Mov. Disord. 22, 399-403. doi: 10.1002/mds. 21309

Dong, J., Beard, J. D., Umbach, D. M., Park, Y., Huang, X., Blair, A., et al. (2014). Dietary fat intake and risk for Parkinson's disease. Mov. Disord. 29, 1623-1630. doi: $10.1002 / \mathrm{mds} .26032$

Dong, M. X., Hu, L., Huang, Y. J., Xu, X. M., Liu, Y., and Wei, Y. D. (2017a). Cerebrovascular risk factors for patients with cerebral watershed infarction: a case-control study based on computed tomography angiography in a population from Southwest China. Medicine 96:e7505. doi: 10.1097/MD.00000000000 07505

Dong, M. X., Hu, Q. C., Shen, P., Pan, J. X., Wei, Y. D., Liu, Y. Y., et al. (2016). Recombinant Tissue Plasminogen Activator Induces Neurological Side Effects Independent on Thrombolysis in Mechanical Animal Models of Focal Cerebral Infarction: a Systematic Review and Meta-Analysis. PLoS ONE 11:e0158848. doi: 10.1371/journal.pone.0158848

Dong, M. X., Li, C. M., Shen, P., Hu, Q. C., Wei, Y. D., Ren, Y. F., et al. (2017b). Recombinant tissue plasminogen activator induces long-term anxietylike behaviors via the ERK1/2-GAD1-GABA cascade in the hippocampus of a rat model. Neuropharmacology 128, 119-131. doi: 10.1016/j.neuropharm.2017. 09.039

Dong, M. X., Xu, X. M., Hu, L., Liu, Y., Huang, Y. J., and Wei, Y. D. (2017c). Serum Butyrylcholinesterase Activity: A Biomarker for Parkinson's Disease and Related Dementia. Biomed. Res. Int. 2017:1524107. doi: 10.1155/2017/1 524107

Goodarzi, Z., Mrklas, K. J., Roberts, D. J., Jette, N., Pringsheim, T., and HolroydLeduc, J. (2016). Detecting depression in Parkinson disease: a systematic review and meta-analysis. Neurology 87, 426-437. doi: 10.1212/WNL.0000000000 002898

Gustafsson, H., Nordstrom, A., and Nordstrom, P. (2015). Depression and subsequent risk of Parkinson disease: a nationwide cohort study. Neurology 84, 2422-2429. doi: 10.1212/WNL.0000000000 001684

Hatano, T., Saiki, S., Okuzumi, A., Mohney, R. P., and Hattori, N. (2016). Identification of novel biomarkers for Parkinson's disease by metabolomic technologies. J. Neurol Neurosurg. Psychiatry 87, 295-301. doi: 10.1136/jnnp-2014-309676

Hochberg, Y., and Benjamini, Y. (1990). More powerful procedures for multiple significance testing. Stat. Med. 9, 811-818. doi: 10.1002/sim.4780090710

Hsu, Y. T., Liao, C. C., Chang, S. N., Yang, Y. W., Tsai, C. H., Chen, T. L., et al. (2015). Increased Risk of Depression in Patients with Parkinson 
Disease: a Nationwide Cohort Study. Am. J. Geriatr. Psychiatry 23, 934-940. doi: 10.1016/j.jagp.2014.10.011

Hu, L., Dong, M. X., Zhao, H., Xu, G. H., and Qin, X. Y. (2016). Fibulin5: a novel biomarker for evaluating severity and predicting prognosis in patients with acute intracerebral haemorrhage. Eur. J. Neurol. 23, 1195-1201. doi: $10.1111 /$ ene. 13013

Huang, T., Rifas-Shiman, S. L., Ertel, K. A., Rich-Edwards, J., Kleinman, K., Gillman, M. W., et al. (2015). Pregnancy hyperglycaemia and risk of prenatal and postpartum depressive symptoms. Paediatr. Perinat. Epidemiol. 29, 281-289. doi: 10.1111/ppe.12199

Hynes, N., Ingham, P., Lim, W., Marshall, C., Massagu,é, J., and Pawson, T. (2013). Signalling change: signal transduction through the decades. Nat. Rev. Mol. Cell Biol. 14, 393-398. doi: 10.1038/nrm3581

Icks, A., Albers, B., Haastert, B., Pechlivanis, S., Bokhof, B., Slomiany, U., et al. (2013). Diabetes incidence does not differ between subjects with and without high depressive symptoms-5-year follow-up results of the Heinz Nixdorf Recall Study. Diabet. Med. 30, 65-69. doi: 10.1111/j.1464-5491.2012. 03724.x

Kalia, L., and Lang, A. (2015). Parkinson's disease. Lancet 386, 896-912. doi: 10.1016/S0140-6736(14)61393-3

Kamburov, A., Cavill, R., Ebbels, T. M., Herwig, R., and Keun, H. C. (2011). Integrated pathway-level analysis of transcriptomics and metabolomics data with IMPaLA. Bioinformatics 27, 2917-2918. doi: 10.1093/bioinformatics/btr499

Kanno, T., Tanaka, A., and Nishizaki, T. (2015). Linoleic acid derivative DCP-LA ameliorates stress-induced depression-related behavior by promoting cell surface 5-HT1A receptor translocation, stimulating serotonin release, and inactivating GSK-3beta. Mol. Neurobiol. 51, 523-532. doi: 10.1007/s12035-014-8718-5

Koponen, H., Kautiainen, H., Leppanen, E., Mantyselka, P., and Vanhala, M. (2015). Association between suicidal behaviour and impaired glucose metabolism in depressive disorders. BMC Psychiatry 15:163. doi: 10.1186/s12888-015-0567-x

Kowal, S. L., Dall, T. M., Chakrabarti, R., Storm, M. V., and Jain, A. (2013). The current and projected economic burden of Parkinson's disease in the United States. Mov. Disord. 28, 311-318. doi: 10.1002/mds. 25292

Levinger, I., Selig, S., Jerums, G., Stewart, A., Gaskin, C. J., and Hare, D. L. (2012). Depressed mood, glycaemic control and functional capacity in overweight/obese men with and without type 2 diabetes. Diabetol. Metab. Syndr. 4:46. doi: 10.1186/1758-5996-4-46

Li, L., Shelton, R. C., Chassan, R. A., Hammond, J. C., Gower, B. A., and Garvey, T. W. (2016). Impact of major depressive disorder on prediabetes by impairing insulin sensitivity. J. Diab. Metab. 7:664. doi: 10.4172/2155-6156.10 00664

Liu, X., Zheng, P., Zhao, X., Zhang, Y., Hu, C., Li, J., et al. (2015). Discovery and validation of plasma biomarkers for major depressive disorder classification based on liquid chromatography-mass spectrometry. J. Proteome Res. 14, 2322-2330. doi: 10.1021/acs.jproteome.5b00144

Lucas, M., Mirzaei, F., O’reilly, E. J., Pan, A., Willett, W. C., Kawachi, I., et al. (2011). Dietary intake of n-3 and n-6 fatty acids and the risk of clinical depression in women: a 10-y prospective follow-up study. Am. J. Clin. Nutr. 93, 1337-1343. doi: 10.3945/ajcn.111.011817

Macleod, A. D., Taylor, K. S., and Counsell, C. E. (2014). Mortality in Parkinson's disease: a systematic review and meta-analysis. Mov. Disord. 29, 1615-1622. doi: $10.1002 / \mathrm{mds} .25898$

Marsh, L. (2013). Depression and Parkinson's disease: current knowledge. Curr. Neurol. Neurosci. Rep. 13:409. doi: 10.1007/s11910-013-0409-5

Mostafa, I., Zhu, N., Yoo, M. J., Balmant, K. M., Misra, B. B., Dufresne, C., et al. (2016). New nodes and edges in the glucosinolate molecular network revealed by proteomics and metabolomics of Arabidopsis myb28/29 and cyp79B2/B3 glucosinolate mutants. J. Proteomics 138, 1-19. doi: 10.1016/j.jprot.2016. 02.012

Oh, J., and Kim, T. S. (2017). Serum lipid levels in depression and suicidality: the Korea National Health and Nutrition Examination Survey (KNHANES) 2014. J. Affect Disord. 213, 51-58. doi: 10.1016/j.jad.2017.02.002

Ong, K. L., Morris, M. J., Mcclelland, R. L., Maniam, J., Allison, M. A., and Rye, K. A. (2016). Lipids, lipoprotein distribution and depressive symptoms: the Multi-Ethnic Study of Atherosclerosis. Trans. Psychiatry 6:e962. doi: $10.1038 /$ tp.2016.232

Pan, J., Liu, H., Zhou, J., Liu, Z., Yang, Y., Peng, Y., et al. (2014). Ipsilateral hippocampal proteomics reveals mitochondrial antioxidative stress impairment in cortical-lesioned chronic mild stressed rats. Curr. Mol. Med. 14, 1186-1196. doi: 10.2174/1566524014666141021143333

Pan, W. C., Kile, M. L., Seow, W. J., Lin, X., Quamruzzaman, Q., Rahman, M., et al. (2013). Genetic susceptible locus in NOTCH2 interacts with arsenic in drinking water on risk of type 2 diabetes. PLOS ONE 8:e70792. doi: 10.1371/journal.pone.0070792

Park, H. E., Kim, J. S., Oh, Y. S., Park, I. S., Park, J. W., Song, I. U., et al. (2016). Autonomic nervous system dysfunction in patients with Parkinson disease having depression. J. Geriatr. Psychiatry Neurol. 29, 11-17. doi: 10.1177/0891988715598234

Persons, J. E., and Fiedorowicz, J. G. (2016). Depression and serum low-density lipoprotein: a systematic review and meta-analysis. J. Affect. Disord 206, 55-67. doi: $10.1016 /$ j.jad.2016.07.033

Ponsen, M., Stoffers, D., Twisk, J., Wolters, E., and Berendse, H. (2009). Hyposmia and executive dysfunction as predictors of future Parkinson's disease: a prospective study. Mov. Disord. 24, 1060-1065. doi: 10.1002/mds.22534

Postuma, R. B., Berg, D., Stern, M., Poewe, W., Olanow, C. W., Oertel, W., et al. (2015). MDS clinical diagnostic criteria for Parkinson's disease. Mov. Disord 30, 1591-1601. doi: 10.1002/mds.26424

Pringsheim, T., Jette, N., Frolkis, A., and Steeves, T. D. (2014). The prevalence of Parkinson's disease: a systematic review and meta-analysis. Mov. Disord 29, 1583-1590. doi: 10.1002/mds.25945

Reijnders, J. S., Ehrt, U., Weber, W. E., Aarsland, D., and Leentjens, A. F. (2008). A systematic review of prevalence studies of depression in Parkinson's disease. Mov. Disord. 23, 183-189:quiz 313. doi: 10.1002/mds.21803

Sakata-Yanagimoto, M., and Chiba, S. (2012). Notch2 and immune function. Curr. Top. Microbiol. Immunol. 360, 151-161. doi: 10.1007/82_2012_235

Sharma, S., Moon, C. S., Khogali, A., Haidous, A., Chabenne, A., Ojo, C., et al. (2013). Biomarkers in Parkinson's disease (recent update). Neurochem. Int. 63, 201-229. doi: 10.1016/j.neuint.2013.06.005

Shen, P., Hu, Q., Dong, M., Bai, S., Liang, Z., Chen, Z., et al. (2017). Venlafaxine exerts antidepressant effects possibly by activating MAPK-ERK1/2 and P13K-AKT pathways in the hippocampus. Behav. Brain Res. 335, 63-70. doi: 10.1016/j.bbr.2017.08.011

Sogawa, K., Takano, S., Iida, F., Satoh, M., Tsuchida, S., Kawashima, Y., et al. (2016). Identification of a novel serum biomarker for pancreatic cancer, C4b-binding protein $\alpha$-chain (C4BPA) by quantitative proteomic analysis using tandem mass tags. Br. J. Cancer 115, 949-956. doi: 10.1038/bjc. 2016.295

Starkstein, S. E., Merello, M., Jorge, R., Brockman, S., Bruce, D., Petracca, G., et al. (2008). A validation study of depressive syndromes in Parkinson's disease. Mov. Disord 23, 538-546. doi: 10.1002/mds.21866

Trezzi, J. P., Galozzi, S., Jaeger, C., Barkovits, K., Brockmann, K., Maetzler, W. et al. (2017). Distinct metabolomic signature in cerebrospinal fluid in early parkinson's disease. Mov. Disord 32, 1401-1408. doi: 10.1002/mds.27132

Wishart, D. S. (2016). Emerging applications of metabolomics in drug discovery and precision medicine. Nat. Rev. Drug Discov. 15, 473-484. doi: $10.1038 /$ nrd.2016.32

Xu, H. B., Zhang, R. F., Luo, D., Zhou, Y., Wang, Y., Fang, L., et al. (2012). Comparative proteomic analysis of plasma from major depressive patients: identification of proteins associated with lipid metabolism and immunoregulation. Int. J. Neuropsychopharmacol. 15, 1413-1425. doi: $10.1017 /$ S1461145712000302

Yu, S., Yang, H., Guo, X., Zheng, L., and Sun, Y. (2017). Metabolic syndrome and depressive symptoms among rural Northeast general population in China. BMC Public Health 17:43. doi: 10.1186/s12889-0163913-0

Zhang, A., Zhou, X., Zhao, H., Zou, S., Ma, C. W., Liu, Q., et al. (2017a). Metabolomics and proteomics technologies to explore the herbal preparation affecting metabolic disorders using high resolution mass spectrometry. Mol. Biosyst. 13, 320-329. doi: 10.1039/c6mb00677a

Zhang, X., Yin, X., Yu, H., Liu, X., Yang, F., Yao, J., et al. (2012). Quantitative proteomic analysis of serum proteins in patients with Parkinson's disease using an isobaric tag for relative and absolute quantification labeling, 
two-dimensional liquid chromatography, and tandem mass spectrometry. Analyst 137, 490-495. doi: 10.1039/C1AN15551B

Zhang, Y., He, J. R., Liang, H. B., Lu, W. J., Yang, G. Y., Liu, J. R., et al. (2017b). Diabetes mellitus is associated with late-onset post-stroke depression. J. Affect. Disord. 221, 222-226. doi: 10.1016/j.jad.2017.06.045

Zhao, P., Li, J., Li, Y., Tian, Y., Yang, L., and Li, S. (2017). Integrating Transcriptomics, Proteomics, and Metabolomics Profiling with System Pharmacology for the Delineation of Long-Term Therapeutic Mechanisms of Bufei Jianpi Formula in Treating COPD. Biomed. Res. Int. 2017:7091087. doi: 10.1155/2017/7091087

Zheng, P., Zeng, B., Zhou, C., Liu, M., Fang, Z., Xu, X., et al. (2016). Gut microbiome remodeling induces depressive-like behaviors through a pathway mediated by the host's metabolism. Mol. Psychiatry 21, 786-796. doi: $10.1038 / \mathrm{mp} .2016 .44$
Conflict of Interest Statement: WC was employed by Shanghai Applied Protein Technology Co., Ltd.

The remaining authors declare that the research was conducted in the absence of any commercial or financial relationships that could be construed as a potential conflict of interest.

Copyright $\odot 2018$ Dong, Feng, Xu, Hu, Liu, Jia, Li, Chen and Wei. This is an open-access article distributed under the terms of the Creative Commons Attribution License (CC BY). The use, distribution or reproduction in other forums is permitted, provided the original author(s) and the copyright owner(s) are credited and that the original publication in this journal is cited, in accordance with accepted academic practice. No use, distribution or reproduction is permitted which does not comply with these terms. 